\title{
ON CONTINUOUS MAPPINGS OF PEANO SPACES
}

\author{
BY \\ TIBOR RADO

\section{INTRODUCTION}

The purpose of this paper is to discuss certain topological questions that arose in the theory of length and area. For an over-all picture the reader may consult Youngs [7] $\left(^{1}\right)$. However, we shall make a few general remarks concerning the origin of the topics discussed in the sequel, in the way of motivation. Let $T_{1}\left(\mathcal{P}_{1}\right)=\mathcal{P}^{*}, T_{2}\left(\mathcal{P}_{2}\right)=\mathrm{P}^{*}$ be a pair of continuous mappings from the Peano spaces $P_{1}, P_{2}$ onto the same Peano space $P^{*}$ (a Peano space is a compact, connected, locally connected metric space). We assume that $\mathbb{P}_{1}, \mathbb{P}_{2}$ are homeomorphic. If there exists a homeomorphism $H\left(\mathbb{P}_{1}\right)=\mathbb{P}_{2}$ such that $T_{1}\left(x_{1}\right)=T_{2} H\left(x_{1}\right)$ for every point $x_{1} \in \mathcal{P}_{1}$ then we shall say that $T_{1}$ and $T_{2}$ are topologically similar, and we shall write $T_{1} \sim T_{2}(t s)$. If for every $\epsilon>0$ there exists a homeomorphism $H_{\epsilon}\left(\mathscr{P}_{1}\right)=\mathscr{P}_{2}$ such that the distance, in $\mathbb{P}^{*}$, of the points $T_{1}\left(x_{1}\right), T_{2} H_{\epsilon}\left(x_{1}\right)$ is less than $\epsilon$ for every choice of the point $x_{1} \in \mathscr{P}_{1}$, then $T_{1}, T_{2}$ will be termed $F$-equivalent (equivalent in the Fréchet sense), and we shall write $T_{1} \sim T_{2}(F)$. For example, if $\mathcal{P}_{1}, \mathbb{P}_{2}$ are both 2-cells, and $\mathcal{P}^{*}$ is a subset of Euclidean three-space, then $T_{1}, T_{2}$ may be considered as representations of surfaces $S_{1}, S_{2}$ respectively. If $T_{1} \sim T_{2}(t s)$, then $T_{2}$ may be derived from $T_{1}$ by a change of parameters, and the surfaces $S_{1}, S_{2}$ are considered as identical. The equivalence $T_{1} \sim T_{2}(F)$ yields a far more comprehensive principle of identification. Its main usefulness lies in the fact that the relation $T_{1} \sim T_{2}(F)$ implies that $A\left(S_{1}\right)=A\left(S_{2}\right)$, where $A$ denotes area in the Lebesgue sense (see, for example, Youngs [7]). Thus if $S_{1}$ is given by a representation $T_{1}$, there may exist more favorable representations $T_{2}$ for $S_{1}$ amongst the solutions $T_{2}$ of the relation $T_{1} \sim T_{2}(F)$. These remarks suggest the importance of methods to derive $F$-equivalent mappings $T_{2}$ to a given mapping $T_{1}$. For conciseness, let us use the term $F$-criterion to refer to any criterion that facilitates the decision whether two given mappings $T_{1}, T_{2}$ are $F$-equivalent or not. The development of efficient $F$-criteria is one of the major topological issues that arise in the theory of length and area.

The case when the initial spaces $\mathbb{P}_{1}, \mathscr{P}_{2}$ are both 2-cells is of prime importance. We shall call this case the 2-cell case. Similarly, the 2-sphere case is the case when $\Phi_{1}, \Phi_{2}$ are both 2 -spheres. A first extremely simple $F$-criterion in the 2-sphere case was proposed by Kerékjártó in 1927 [2]. Let $P_{1}, P_{2}$ be 2-spheres, let $M_{1}\left(\mathscr{P}_{1}\right)=\mathfrak{M}, M_{2}\left(\mathscr{P}_{2}\right)=\mathfrak{M}$ be continuous monotone mappings

Presented to the Society, September 17, 1945; received by the editors May 10, 1945.

(') Numbers in square brackets refer to the bibliography. 
from $\mathscr{P}_{1}, \mathbb{P}_{2}$ onto the same Peano space $\mathfrak{M}$, and let $L(\mathfrak{M})$ be a continuous light mapping from $\mathfrak{M}$ onto a subset $\mathbb{P}^{*}$ of Euclidean three-space. (See Whyburn [6] for terminology.) According to Kerékjártó [2], the mappings $T_{1}=L M_{1}$, $T_{2}=L M_{2}$ should then be $F$-equivalent (products of mappings are to be read from the right to the left). In 1935, Morrey [3] made, apparently independently, the same assertion in the 2-cell case, and proceeded to make extensive applications to surface area. In 1940, Youngs discovered that the $F$-criteria proposed by Kerékjártó and Morrey were both inadequate, and began comprehensive studies to develop a satisfactory topological foundation for the theory of surface area. In an important paper, published recently [7], Youngs developed a new $F$-criterion, and derived various fundamental applications. This paper of Youngs is concerned with the 2-sphere case. The following metric results of Youngs are relevant for the purposes of these introductory remarks. Let $T(\mathcal{P})=\mathbb{P}^{*}$ be a continuous mapping from the 2 -sphere $\mathscr{P}$ onto a subset $\mathbb{P}^{*}$ of Euclidean three-space. Let $T=L M, M(\mathbb{P})=\mathfrak{M}, L(\mathfrak{M})=\mathbb{P}^{*}$ be a monotone-light factorization of $T$ (see Whyburn [6]), and let us suppose that the middle-space $\mathfrak{M}$ is not a dendrite. Let then $\Gamma^{1}, \ldots, \Gamma^{n}, \cdots$ be the proper cyclic elements of $\mathfrak{M}$, and for each $n$, let $\alpha^{n}(\mathfrak{M})=\Gamma^{n}$ be the (unique) monotone retraction from $\mathfrak{M}$ onto $\Gamma^{n}$ (see Whyburn [6]). Let us put $T^{n}=L \alpha^{n} M$. In the sequel, the mappings $T^{1}, \ldots, T^{n}, \cdots$ will be said to constitute the cyclic decomposition of $T$. Let $A(T), A\left(T^{1}\right), \cdots$ denote the Lebesgue areas of the surfaces determined by the representations $T, T^{1}, \cdots$. According to Youngs, we have then the fundamental formula $A(T)=A\left(T^{1}\right)$ $+\cdots+A\left(T^{n}\right)+\cdots$. According to the terminology used in the sequel, this result states the fact that the Lebesgue area $A$ is cyclicly additive. Now suppose we are given two continuous mappings $T_{1}\left(\mathscr{P}_{1}\right)=\mathbb{P}^{*}, T_{2}\left(\mathcal{P}_{2}\right)=\mathbb{P}^{*}$ from the 2-spheres $\mathbb{P}_{1}, \mathbb{P}_{2}$ onto the same subset $\mathbb{P}^{*}$ of Euclidean three space. Let us assume that $T_{1}$ and $T_{2}$ are related in the manner considered by Kerékjártó (see above), and let us express this assumption by writing $T_{1} \sim T_{2}(K)\left(T_{1}\right.$ and $I_{2}$ are equivalent in the Kerékjártó sense). Even though the relation $T_{1} \sim T_{2}(F)$ is not implied by this assumption, Youngs shows that $A\left(T_{1}\right)$ $=A\left(T_{2}\right)$, a most remarkable result. In view of these significant developments, the present writer undertook a further application of the results of Youngs. The Lebesgue area $A(T)$ has the character of an upper area. An entirely different type of definition, whose basic idea is due to Geöcze, yields a lower area $a(T)$ (see [5]). Combining the methods of Youngs with analytic methods available in surface area theory, the writer succeeded in obtaining a partial solution of a fundamental problem already suggested by Geöcze himself, by showing that $a(T)=A(T)$ whenever $A(T)<+\infty$, and that $a(T)>0$ if $A(T)=+\infty$.

These results are relative to the 2 -sphere case. Analogous metric results for the 2-cell case, which is of greater importance at present, were derived by the present writer in a rather devious manner, by means of a reduction 
to the 2-sphere case. This method involved a great deal of cumbersome ad hoc metric and topological detail, and thus an attempt to develop a direct approach for the 2-cell case seemed desirable. Consequently, the writer attempted to extend the topological results of Youngs to the 2-cell case. As a matter of fact, Youngs himself pointed out various new difficulties that arise in this case. Correspondence with Youngs (engaged in war work overseas) revealed that ultimate clarification of the topology of the 2-cell case, to an extent comparable with the 2 -sphere case, may require considerable time and effort. On the other hand, the progress achieved in recent years in the analytic foundations of the theory of surface area seemed to justify the hope that the relevant metric results could be obtained on the basis of greatly reduced topological requirements. This is indeed the case, and the purpose of this paper is to give a discussion of the topological results involved in this approach. Part 1 is devoted to a study of the cyclic decomposition of continuous mappings of general Peano spaces. Parts 2 and 3 are concerned with special results concerning mappings of 2 -spheres and 2-cells. The extension of these results to $n$-cells and $n$-spheres may lead to problems of considerable interest and difficulty. Part 4 contains a general theorem on cyclic additivity that covers both the Lebesgue area $A(T)$ and the lower area $a(T)$. For the Lebesgue area $A(T)$ in the 2-cell case, a theorem of this type has been stated first by Morrey [4]. His proof makes extensive use of the fact that $A(T)$ is $F$-invariant (that is, $T_{1} \sim T_{2}(F)$ implies that $A\left(T_{1}\right)=A\left(T_{2}\right)$ ), and also of the inadequate $F$-criterion proposed by him. Thus a thorough revision of the proof is necessary, and the present lack of an adequate $F$-criterion for the 2-cell case makes this revision an interesting undertaking. As a matter of fact, it turns out that no $F$-criterion is needed as far as the cyclic additivity theorem is concerned. We prove the theorem for a general function $F(T)$ of the mapping $T$ without making any assumption concerning $F$-invariance. The theorem applies both to the Lebesgue area $A(T)$ and the lower area $a(T)$, and covers simultaneously the 2 -sphere case and the 2 -cell case. In fact, the initial space is required only to possess a certain property shared by 2-cells and 2 -spheres, but the writer was unable to determine the precise scope of this property $\left({ }^{2}\right)$. The assumptions concerning $F(T)$ were chosen, of course, with regard to present applications, and considerable simplifications may be possible both in the proof and in the choice of the assumptions. To facilitate such improvements, the mutual implications of the assumptions used are discussed in some detail.

The following remarks may be helpful as regards notations and terminology. All spaces used are metric spaces. $\rho\left(p_{1}, p_{2}\right)$ denotes the distance of the points $p_{1}, p_{2} ; d(E)$ denotes the diameter of the set $E$. Even though up to five spaces will be considered simultaneously at times, we agree to use $\rho$ and $d$

(2) This point has been cleared up by G. T. Whyburn. Cf. footnote $3, \S 4.12$. 
without indicating the space involved in the notation itself, since it will be clear from the context just what space is meant. For brevity, we shall use the terms 2-cell and 2-sphere to refer to topological 2-cells and topological 2 -spheres. In connection with the cyclic additivity theorem, the following remarks will be used to insure clarity and conciseness. Let $K$ be a class of elements to be denoted by $a, b, c, \cdots$. Let $a_{1}, \cdots, a_{n}, \cdots$ be a (finite or countably infinite) sequence of elements of $K$. An element $a \in K$ may occur in the sequence not at all, or a finite number of times, or an infinite number of times. In several instances, it will be immaterial in what order the terms of the sequence are listed, so long as the number of occurrences of each element of $K$ in the sequence remains the same. To indicate this fact, we shall put the sequence $a_{1}, \cdots, a_{n}, \cdots$ between square brackets: $\left[a_{1}, \cdots, a_{n}, \cdots\right]$. Thus an equation $\left[a_{n}\right]=\left[b_{n}\right]$ means that the sequence $b_{n}$ is a rearrangement of the sequence $a_{n}$, and it does not mean that $a_{n}=b_{n}$ for every $n$. An equation $\left[a_{n}\right]=\left[b_{n}\right]+\left[c_{n}\right]+\cdots$ will mean that the sequence $a_{n}$ is the result of dovetailing the sequences $b_{n}, c_{n}, \cdots$. Let now $f(a)$ be a real-valued, non-negative function defined on $K$ that may take on the value $+\infty$. Then the symbol $f\left(\left[a_{n}\right]\right)$ will denote the sum of the series $f\left(a_{1}\right)+\cdots+f\left(a_{n}\right)+\cdots$ if this series is convergent, and $+\infty$ if the series is divergent (the latter case is meant to include the case when $f\left(a_{n}\right)=+\infty$ for one or more values of $\left.n\right)$. Since $f$ is non-negative, clearly $\left[a_{n}\right]=\left[b_{n}\right]$ implies $f\left(\left[a_{n}\right]\right)=f\left(\left[b_{n}\right]\right)$, and $\left[a_{n}\right]=\left[b_{n}\right]$ $+\left[c_{n}\right]+\cdots$ implies $f\left(\left[a_{n}\right]\right)=f\left(\left[b_{n}\right]\right)+f\left(\left[c_{n}\right]\right)+\cdots$. It will be convenient to consider empty sequences. If $\sigma$ is an empty sequence, then we agree to put $f([\sigma])=0$.

Extensive use will be made of the topology of Peano spaces, and in particular of cyclic element theory. For all conceptions and facts used without explanation, the reader is referred to Whyburn [6]. Some of the applications of the results of this paper are stated in a recent note by the writer [5]. Let us mention explicitly a result not included in [5]. This result states that the Lebesgue area $A(T)$ and the lower area $a(T)$ are $K$-invariant in both the 2-sphere case and the 2-cell case (that is, $T_{1} \sim T_{2}(K)$ implies $A\left(T_{1}\right)=A\left(T_{2}\right)$, $\left.a\left(T_{1}\right)=a\left(T_{2}\right)\right)$. As regards $A$ in the 2 -sphere case, the $K$-invariance has been already established by Youngs. As regards the 2-cell case, let us note that the topological results must be supplemented by a considerable amount of analytic results to secure the theorem just stated.

\section{F-equivalent mappings of Peano spaces}

1.1. Let us first recall some general facts concerning continuous mappings. Let $T(P)=P^{*}$ be a continuous mapping from the Peano space $P$ onto the Peano space $P^{*}$ (some of the facts stated in the sequel obviously hold for more general spaces). Then $T$ is uniformly continuous on $P$. That is, for every $\epsilon>0$ there exists a $\delta>0$, such that the conditions $p_{1}, p_{2} \in P, \rho\left(p_{1}, p_{2}\right)<\delta$ imply $\rho\left[T\left(p_{1}\right), T\left(p_{2}\right)\right]<\epsilon$. Let now $T_{n}(P)=P^{*}$ be a sequence of continuous 
mappings. Then the sequence $T_{n}(P)$ is termed equicontinuous on $P$ if for every $\epsilon>0$ there exists a $\delta>0$, such that for every $n$ and every pair of points $p_{1}, p_{2} \in P$ the inequality $\rho\left(p_{1}, p_{2}\right)<\delta$ implies $\rho\left[T_{n}\left(p_{1}\right), T_{n}\left(p_{2}\right)\right]<\epsilon$.

Let $T_{n}(P)=P^{*}$ be a sequence of continuous mappings and $T(P)=P^{*}$ be a continuous mapping. If for every $\epsilon>0$ there exists an $N$ such that $\rho\left[T_{n}(p), T(p)\right]<\epsilon$ for $n>N$ and for every point $p \in P$, then the sequence $T_{n}$ is said to converge uniformly to $T$ on $P$. If $T_{n}$ converges to $T$ uniformly on $P$, then it follows easily that the sequence $T_{n}$ is equicontinuous on $P$.

Let $T_{n}(P)=P^{*}$ be a sequence of continuous mappings. If this sequence is equicontinuous on $P$, then it contains a uniformly convergent subsequence. That is, there exists an infinite sequence of positive integers $n_{1}<n_{2}<\ldots$ $<n_{k}<\cdots$, such that $T_{n_{k}}, k=1,2, \cdots$, converges on $P$ uniformly to a continuous mapping $T(P)=P^{*}$ (cf. Hahn $[1$, p. 231, Theorem 29.4.1]).

1.2. Continuation. Let $T_{n}(P)=P^{*}$ be a sequence of continuous mappings converging on $P$ uniformly to a continuous mapping $T(P)=P^{*}$. Suppose that each $T_{n}$ is monotone (that is, $T^{-1}\left(p^{*}\right)$ is a continuum for every point $p^{*} \in P^{*}$ ). Then the limit mapping $T$ is also monotone (see Whyburn [6, p. 174, Theorem 3.11]).

1.3. Let $T(\mathscr{P})=\Phi^{*}$ be a continuous mapping, where $\mathcal{P}, \mathscr{P}^{*}$ are Peano spaces. For each point $p^{*} \in \mathcal{P}^{*}$, the set $T^{-1}\left(p^{*}\right)$ is a non-empty closed subset of $\mathcal{P}$, and hence every component of $T^{-1}\left(p^{*}\right)$ is a continuum (possibly a single point). We shall denote by $K(T)$ the collection of all the components of all the sets $T^{-1}\left(p^{*}\right), p^{*} \in \mathcal{P}^{*}$. The symbol $\gamma \in K(T)$ will mean that the continuum $\gamma$ is a member of the collection $K(T)$. Clearly, if $\gamma_{1} \in K(T), \gamma_{2} \in K(T)$, and $\gamma_{1} \neq \gamma_{2}$ then $\gamma_{1} \gamma_{2}=0$. Furthermore, $\mathcal{P}=\sum \gamma, \gamma \in K(T)$. The collection $K(T)$ possesses a number of important properties and is fundamental in the study of the mapping $T$ (see Whyburn [6, chap. 7]). A continuum $\gamma \in K(T)$ will be termed a maximal model continuum (under $T$ in $P$ ).

1.4. Given $T(\mathscr{P})=\mathbb{P}^{*}$ as in 1.3 , there exists a factorization of $T$ of the form $T=L M, M(\mathscr{P})=\mathfrak{M}, L(\mathfrak{M})=\mathbb{P}^{*}$, where $M$ is monotone and $L$ is light. That is, for every point $p^{*} \in \mathbb{P}^{*}, L^{-1}\left(p^{*}\right)$ is a totally disconnected subset of $\mathfrak{M}$, and for every point $\bar{a} \in \mathfrak{M}, M^{-1}(\bar{a})$ is a continuum in $P$ (see Whyburn [6, p. 141, Theorem 4.1]). The monotone factor $M$ satisfies the relation $K(T)=K(M)$. In other words, $T$ and its monotone factor $M$ determine the same collection of maximal model continua in $P$ (cf. Whyburn, loc. cit.). The space $\mathfrak{M}$ will be termed the middle-space.

1.5. Continuation. Let $T=L_{*} M_{*}, M_{*}(\mathscr{P})=\mathfrak{M}_{*}, L_{*}\left(\mathfrak{M}_{*}\right)=\mathbb{P}^{*}$ be a second monotone-light factorization of $T$. Then $K\left(M_{*}\right)=K(T)=K(M)$ (cf. 1.4). It follows that for every point $\bar{a} \in \mathfrak{M}$ the set $M_{*} M^{-1}(\bar{a})$ reduces to a single point in $\mathfrak{M}_{*}$, and for every point $\bar{a}_{*} \in \mathfrak{M}_{*}$ the set $M M_{*}^{-1}\left(\bar{a}_{*}\right)$ reduces to a single point in $\mathfrak{M}$. We obtain in this manner two single-valued mappings $h=M_{*} M^{-1}$ and $h_{*}=M M_{*}^{-1}$. The following facts are readily established (see Whyburn, loc. cit.): 
(i) $h$ and $h_{*}$ are both topological mappings, and $h^{-1}=h_{*}, h(\mathfrak{M})=\mathfrak{M}_{*}$, $h_{*}\left(\mathfrak{M}_{*}\right)=\mathfrak{M}$.

(ii) $L(\bar{a})=L_{*} h(\bar{a})$ for every point $\bar{a} \in \mathfrak{M}$.

(iii) $M_{*}(p)=h M(p)$ for every point $p \in \mathcal{P}$.

In particular, it follows that the middle-spaces $\mathfrak{M}$ and $\mathfrak{M}_{*}$ are homeomorphic. It follows easily that (iii) determines the topological mapping $h(\mathfrak{M})=\mathfrak{M}_{*}$ univocally.

1.6. Let $P, P^{*}, \Phi$ be Peano spaces. Let $L\left(P^{*}\right)=\Phi$ be a continuous light mapping, and let $T^{n}(P)=P^{*}$ be a sequence of continuous mappings. Suppose that the sequence of mappings $L T^{n}$ is equicontinuous on $P$. Then the sequence $T^{n}$ is also equicontinuous on $P$ (cf. 1.1).

Proof. Let $\epsilon>0$. Since $L$ is light, there exists a $\sigma>0$ such that

$$
d\left(C^{*}\right)<\epsilon \text { if } d\left[L\left(C^{*}\right)\right]<\sigma,
$$

where $C^{*}$ is a generic notation for a continuum in $P^{*}$ (this is an immediate consequence of Whyburn [6, p. 131, Theorem 4.41]). Since the sequence $L T^{n}$ is equicontinuous, there exists an $\eta>0$ such that

$$
\rho\left[L T^{n}\left(q_{1}\right), L T^{n}\left(q_{2}\right)\right]<\sigma \quad \text { if } q_{1}, q_{2} \in P, \rho\left(q_{1}, q_{2}\right)<\eta .
$$

As a consequence, we have

$$
d\left[L T^{n}(C)\right]<\sigma \quad \text { if } \quad d(C)<\eta,
$$

where $C$ is a generic notation for a continuum in $P$. Since $P$ is a Peano space, we have a $\delta>0$ such that the conditions $p_{1}, p_{2} \in P, \rho\left(p_{1}, p_{2}\right)<\delta$ imply the existence of a continuum $C$ such that $p_{1}+p_{2} \subset C \subset P, d(C)<\eta$ (see Whyburn $[6$, p. 22, Theorem 15.5$])$. Now take any two points

$$
x_{1}, x_{2} \in P, \quad \rho\left(x_{1}, x_{2}\right)<\delta .
$$

By the choice of $\delta$, we have then a continuum $C$ such that

$$
x_{1}+x_{2} \subset C \subset P, d(C)<\eta \text {. }
$$

In view of (3), we obtain from (5)

$$
\begin{gathered}
\rho\left[T^{n}\left(x_{1}\right), T^{n}\left(x_{2}\right)\right] \leqq d\left[T^{n}(C)\right], \\
d\left[L T^{n}(C)\right]<\sigma .
\end{gathered}
$$

Now $T^{n}(C)$ is a continuum in $P^{*}$, and hence (7) and (1) yield

$$
d\left[T^{n}(C)\right]<\epsilon .
$$

(4), (6) and (8) yield

$$
\rho\left[T^{n}\left(x_{1}\right), T^{n}\left(x_{2}\right)\right]<\epsilon \quad \text { if } x_{1}, x_{2} \in P, \rho\left(x_{1}, x_{2}\right)<\delta .
$$

Since $\delta$ is independent of $x_{1}, x_{2}, n$, the equicontinuity of the sequence $T^{n}$ is established. 
1.7. Let us now consider a pair of continuous mappings $T_{1}\left(P_{1}\right)=\mathscr{P}^{*}$, $T_{2}\left(\mathscr{P}_{2}\right)=\mathcal{P}^{*}$, where $\mathcal{P}_{1}, \mathcal{P}_{2}$ are Peano spaces, and $T_{1} \sim T_{2}(F)$. By definition (see the introduction), for every $\epsilon>0$ we have then a topological mapping $H_{\epsilon}\left(P_{1}\right)=\mathscr{P}_{2}$ such that $\rho\left[T_{1}\left(x_{1}\right), T_{2} H_{\epsilon}\left(x_{1}\right)\right]<\epsilon$ for every point $x_{1} \in \mathcal{P}_{1}$. There follows the existence of a sequence of topological mappings $H_{n}\left(\Phi_{1}\right)=\Phi_{2}$ such that on setting

$$
\lambda_{n}=\max \rho\left[T_{1}\left(x_{1}\right), T_{2} H_{n}\left(x_{1}\right)\right], \quad x_{1} \in \mathcal{P}_{1},
$$

we have $\lambda_{n} \rightarrow 0$. In other words, the sequence $T_{2} H_{n}$ converges uniformly to $T_{1}$. As a consequence, the sequence $T_{2} H_{n}$ is equicontinuous on $P_{1}$ (see 1.1). Now let

$$
\begin{array}{lll}
T_{1}=L_{1} M_{1}, & M_{1}\left(\mathscr{P}_{1}\right)=\mathfrak{M}_{1}, & L_{1}\left(\mathfrak{M}_{1}\right)=\mathbb{P}^{*}, \\
T_{2}=L_{2} M_{2}, & M_{2}\left(\mathcal{P}_{2}\right)=\mathfrak{M}_{2}, & L_{2}\left(\mathfrak{M}_{2}\right)=\mathbb{P}^{*}
\end{array}
$$

be arbitrarily chosen monotone-light factorizations for $T_{1}, T_{2}$ respectively. Then $T_{2} H_{n}=L_{2} M_{2} H_{n}=L_{2} T^{n}$, where $T^{n}=M_{2} H_{n}$. Since the sequence $T_{2} H_{n}$ $=L_{2} T^{n}$ is equicontinuous and $L_{2}$ is light, it follows by 1.6 that the sequence $T^{n}=M_{2} H_{n}$ is also equicontinuous on $P_{1}$. By 1.1 the sequence $M_{2} H_{n}$ contains therefore a uniformly convergent subsequence $M_{2} H_{k_{n}}, k_{1}<k_{2}<\cdots<k_{n}<\cdots$. Replacing the original sequence $H_{n}$ by the subsequence $H_{k_{n}}$ we see that we can assume without loss of generality that the initially chosen sequence $H_{n}$ is such that the sequence $M_{2} H_{n}$ is uniformly convergent on $P_{1}$. Since clearly each $M_{2} H_{n}$ is a continuous monotone mapping from $\mathbb{P}_{1}$ onto $\mathfrak{M}_{2}$, the limit mapping, to be denoted by $\bar{M}_{1}$, is also a continuous monotone mapping from $\mathbb{P}_{1}$ onto $\mathfrak{M}_{2}$ (see 1.2 ). Now since $L_{2}$ is uniformly continuous on $\mathfrak{M}_{2}$ and $M_{2} H_{n}$ converges uniformly to $\bar{M}_{1}$ on $\Phi_{1}$, it follows that $T_{2} H_{n}=L_{2} M_{2} H_{n}$ converges uniformly to $L_{2} \bar{M}_{1}$ on $P_{1}$. But $T_{2} H_{n}$ converges uniformly to $T_{1}$ as we noted above. Thus $T_{1}=L_{2} \bar{M}_{1}$. In other words, we have for $T_{1}$ the monotone-light factorization

$$
T_{1}=L_{2} \bar{M}_{1}, \quad \bar{M}_{1}\left(\mathscr{P}_{1}\right)=\mathfrak{M}_{2}, \quad L_{2}\left(\mathfrak{M}_{2}\right)=\mathbb{P}^{*} .
$$

Comparison of the factorizations (1) and (3) yields, by 1.4, 1.5, the existence of a topological mapping $h\left(\mathfrak{M}_{1}\right)=\mathfrak{M}_{2}$ such that

$$
L_{1}=L_{2} h, \quad \bar{M}_{1}=h M_{1} .
$$

In particular, it follows that the middle-spaces $\mathfrak{M}_{1}, \mathfrak{M}_{2}$ are homeomorphic.

Now let $Z_{1}$ denote any continuous mapping from $\mathfrak{M}_{1}$ into $\mathfrak{M}_{1}$, that is, $Z_{1}\left(\mathfrak{M}_{1}\right) \subset \mathfrak{M}_{1}$. We assert that

$$
L_{1} Z_{1} M_{1} \sim L_{2} h Z_{1} h^{-1} M_{2}(F) .
$$

Indeed, let us put for brevity

$$
X_{2}=L_{2} h Z_{1} h^{-1}, \quad T_{1}^{*}=L_{1} Z_{1} M_{1}, \quad T_{2}^{*}=L_{2} h Z_{1} h^{-1} M_{2} .
$$


We have then, in view of (4), (5),

$$
\begin{aligned}
& T_{1}^{*}=L_{1} Z_{1} M_{1}=L_{2} h Z_{1} h^{-1} h M_{1}=X_{2} \bar{M}_{1}, \\
& T_{2}^{*} H_{n}=L_{2} h Z_{1} h^{-1} M_{2} H_{n}=X_{2} M_{2} H_{n} .
\end{aligned}
$$

Now $X_{2}$ is uniformly continuous on $\mathfrak{M}_{2}$ and $M_{2} H_{n}$ converges to $\bar{M}_{1}$ uniformly on $P_{1}$. Hence (7) and (8) yield the result that $T_{2}{ }^{*} H_{n}$ converges to $T_{1}{ }^{*}$ uniformly on $P_{1}$. Hence, for given $\epsilon>0$, there exists a positive integer $n(\epsilon)$ such that

$$
\rho\left[T_{1}^{*}\left(x_{1}\right), T_{2}^{*} H_{n(\epsilon)}\left(x_{1}\right)\right]<\epsilon
$$

for every point $x_{1} \in \mathcal{P}_{1}$. Since $H_{n(\epsilon)}$ is a topological mapping from $\mathbb{P}_{1}$ onto $\mathbb{P}_{2}$ and $\epsilon$ was arbitrary, it follows that $T_{1}{ }^{*} \sim T_{2}{ }^{*}(F)$, and thus (5) is proved. The results obtained will be summarized in the next section.

1.8. Theorem. Let $T_{1}\left(\mathscr{P}_{1}\right)=\mathbb{P}^{*}, T_{2}\left(\mathscr{P}_{2}\right)=\mathbb{P}^{*}$, where $\mathbb{P}_{1}, \mathbb{P}_{2}$ are Peano spaces, be continuous mappings such that $T_{1} \sim T_{2}(F)$. Let

$$
\begin{array}{lll}
T_{1}=L_{1} M_{1}, & M_{1}\left(\mathscr{P}_{1}\right)=\mathfrak{M}_{1}, & L_{1}\left(\mathfrak{M}_{1}\right)=\mathbb{P}^{*}, \\
T_{2}=L_{2} M_{2}, & M_{2}\left(\mathbb{P}_{2}\right)=\mathfrak{M}_{2}, & L_{2}\left(\mathfrak{M}_{2}\right)=\mathbb{P}^{*}
\end{array}
$$

be arbitrarily chosen monotone-light factorizations for $T_{1}, T_{2}$ respectively. Then the middle-spaces $\mathfrak{M}_{1}, \mathfrak{M}_{2}$ are homeomorphic, and there exists a topological mapping $h\left(\mathfrak{M}_{1}\right)=\mathfrak{M}_{2}$ with the following properties.

(i) $L_{1}\left(\bar{a}_{1}\right)=L_{2} h\left(\bar{a}_{1}\right)$ for every point $\bar{a}_{1} \in \mathfrak{M}_{1}$.

(ii) If $Z_{1}$ is any continuous mapping from $\mathfrak{M}_{1}$ into $\mathfrak{M}_{1}$ (that is, $\left.Z_{1}\left(\mathfrak{M}_{1}\right) \subset \mathfrak{M}_{1}\right)$, then $L_{1} Z_{1} M_{1} \sim L_{2} h Z_{1} h^{-1} M_{2}(F)$.

Indeed, the topological mapping $h\left(\mathfrak{M}_{1}\right)=\mathfrak{M}_{2}$ of 1.7 possesses the properties (i) and (ii) in view of $1.7(4), 1.7$ (5). Let us note that the existence of a topological mapping $h\left(\mathfrak{M}_{1}\right)=\mathfrak{M}_{2}$ with the property (i) has been already established by Youngs (see [7, pp. 771-775]). In fact, Youngs proved a great deal more than (i), but it seems that the property (ii) has not been considered previously. Roughly speaking, our theorem states the fact that the $F$-equivalence of $T_{1}, T_{2}$ is inherited by all the partial mappings of $T_{1}, T_{2}$, if these partial mappings are paired off in the manner indicated in the statement of property (ii). Note that on choosing $Z_{1}$ as the identity transformation on $\mathfrak{M}_{1}$, (ii) yields $T_{1} \sim T_{2}(F)$. Thus the existence of a topological mapping $h\left(\mathfrak{M}_{1}\right)=\mathfrak{M}_{2}$ with the property (ii) is a necessary and (trivially) sufficient condition for the relation $T_{1} \sim T_{2}(F)$. On the other hand, let us note explicitly that property (i) does not imply property (ii) as simple examples show. Let us also observe that our proof of the theorem stated in this section has many features in common with that of Youngs, loc. cit.; the main difference lies perhaps in the fact that we have reduced, in a sense, the study of two monotone-light factorizations of two $F$-equivalent mappings $T_{1}, T_{2}$ to the study of two monotone-light fac- 
torizations of the same mapping $T_{1}$. Furthermore, it would seem that our reliance on general theorems concerning equicontinuous sequences eliminates a substantial amount of $\epsilon, \delta$ type argument.

1.9. Let $T(\mathscr{P})=\mathscr{P}^{*}$, where $\mathcal{P}$ is a Peano space, be a continuous mapping. We define the cyclic decumposition of $T$ as follows. Let

$$
T=L M, \quad M(\mathscr{P})=\mathfrak{M}, \quad L(\mathfrak{M})=\mathscr{P}^{*}
$$

be a monotone-light factorization of $T$. Assume first that the middle space $\mathfrak{M}$ is not a dendrite, and let $\Gamma^{1}, \cdots, \Gamma^{n}, \cdots$ be the sequence of the proper cyclic elements of $\mathfrak{M}$. Let us recall that a Peano space has at most a countably infinite number of proper cyclic elements (see Whyburn [6, p. 71, Theorem $4.2]$ ). Of course, the sequence $\Gamma^{n}$ may be finite, but it. has at least one element since $\mathfrak{M}$ is not a dendrite. Now for each $\Gamma^{n}$ there exists a unique monotone retraction $\mu^{n}(\mathfrak{M})=\Gamma^{n}$ (see Whyburn [6, p. 143, Theorem 5.1]). The cyclic decomposition $\Delta(T)$ of $T$ is defined then by the formula

$$
\Delta(T)=\left[L \mu^{1} M, \cdots, L \mu^{n} M, \cdots\right],
$$

where the square brackets enclosing the sequence $L \mu^{1} M, \cdots, L \mu^{n} M, \cdots$ are used in the sense explained in the introduction. The mappings $L \mu^{n} M$ will be termed the cyclic partial mappings of $T$.

The preceding definition presupposes that $\mathfrak{M}$ is not a dendrite. If $\mathfrak{M}$ is a dendrite, then we define $\Delta(T)$ as an empty collection of mappings, in symbols $\Delta(T)=0$.

In defining $\Delta(T)$, we used a definite factorization of $T$. We proceed to show that $\Delta(T)$ is independent of the choice of this factorization.

1.10. Let us consider two monotone-light factorizations

$$
\begin{array}{lll}
T=L_{1} M_{1}, & M_{1}(\mathscr{P})=\mathfrak{M}_{1}, & L_{1}\left(\mathfrak{M}_{1}\right)=\mathscr{P}^{*}, \\
T=L_{2} M_{2}, & M_{2}(\mathscr{P})=\mathfrak{M}_{2}, & L_{2}\left(\mathfrak{M}_{2}\right)=\mathscr{P}^{*}
\end{array}
$$

of $T$. Then there exists a topological mapping $h\left(\mathfrak{M}_{1}\right)=\mathfrak{M}_{2}$ such that $L_{1}=L_{2} h$, $M_{2}=h M_{1}$ (see 1.4,1.5). If $\mathfrak{M}_{1}$ is a dendrite, then $\mathfrak{M}_{2}$ is also a dendrite (since $\mathfrak{M}_{1}, \mathfrak{M}_{2}$ are homeomorphic), and hence in this case there exist no cyclic partial mappings of $T$. Let us next assume that $\mathfrak{M}_{1}$ is not a dendrite, and let $\Gamma_{1}^{1}, \cdots, \Gamma_{1}^{n}, \cdots$ be the proper cyclic elements of $\mathfrak{M}_{1}$. In view of the homeomorphism $h\left(\mathfrak{M}_{1}\right)=\mathfrak{M}_{2}$, it follows that the sets $\Gamma_{2}^{n}=h\left(\Gamma_{1}^{n}\right), n=1,2, \cdots$, coincide with the proper cyclic elements of $\mathfrak{M}_{2}$. If $\mu_{i}^{n}\left(\mathfrak{M}_{i}\right)=\Gamma_{i}^{n}$ is the unique monotone retraction from $\mathfrak{M}_{i}$ onto $\Gamma_{i}^{n}, i=1,2$, then clearly $h \mu_{1}^{n} h^{-1}$ is a monotone retraction from $\mathfrak{M}_{2}$ onto $\Gamma_{2}^{n}$. Since there exists just one such monotone retraction, it follows that $\mu_{2}^{n}=h \mu_{1}^{n} h^{-1}$. Hence $L_{2} \mu_{2}^{n} M_{2}=L_{2} h \mu_{1}^{n} h^{-1} M_{2}=L_{1} \mu_{1}^{n} M_{1}$. In other words, the sequences of cyclic partial mappings

$$
L_{1} \mu_{1}^{1} M_{1}, \cdots, L_{1} \mu_{1}^{n} M_{1}, \cdots, \quad L_{2} \mu_{2}^{1} M_{2}, \cdots, L_{2} \mu_{2}^{n} M_{2}, \cdots
$$


agree term for term. Thus the factorizations (1), (2) yield the same cyclic decomposition for $T$. Hence $\Delta(T)$ depends solely upon $T$, and is independent of the choice of the monotone-light factorization for $T$.

1.11. Given two continuous mappings $T_{1}\left(\mathscr{P}_{1}\right)=\Phi^{*}, T_{2}^{\prime}\left(\mathscr{P}_{2}\right)=\Phi^{*}$, where $\Phi_{1}, \Phi_{2}$ are Peano spaces, we shall say that their cyclic decompositions $\Delta\left(T_{1}\right)$, $\Delta\left(T_{2}\right)$ are $F$-equivalent if one of the following two conditions holds.

(i) $\Delta\left(T_{1}\right)$ and $\Delta\left(T_{2}\right)$ both reduce to zero.

(ii) $0 \neq \Delta\left(T_{1}\right)=\left[T_{1}^{1}, \cdots, T_{1}^{n}, \cdots\right], 0 \neq \Delta\left(T_{2}\right)=\left[T_{2}^{1}, \cdots, T_{2}^{n}, \cdots\right]$, and there exists a rearrangement $\bar{T}_{2}^{1}, \cdots, \bar{T}_{2}^{n}, \cdots$ of the sequence $T_{2}^{1}, \cdots$, $T_{2}^{n}, \ldots$ such that $T_{1}^{n} \sim \bar{T}_{2}^{n}(F)$ for every $n$. This requirement implies that the collections $\Delta\left(T_{1}\right), \Delta\left(T_{2}\right)$ are both finite or both infinite (see the introduction).

We shall write $\Delta\left(T_{1}\right) \sim \Delta\left(T_{2}\right)(F)$ to state the fact that $\Delta\left(T_{1}\right), \Delta\left(T_{2}\right)$ are $F$-equivalent in the sense of the preceding definition. Using this terminology, we proceed to establish the following result.

THEOREM. If $T_{1} \sim T_{2}(F)$, then $\Delta\left(T_{1}\right) \sim \Delta\left(T_{2}\right)(F)$.

Proof. Let us choose monotone-light factorizations

$$
\begin{array}{lll}
T_{1}=L_{1} M_{1}, & M_{1}\left(\mathcal{P}_{1}\right)=\mathfrak{M}_{1}, & L_{1}\left(\mathfrak{M}_{1}\right)=\mathcal{P}^{*}, \\
T_{2}=L_{2} M_{2}, & M_{2}\left(\mathscr{P}_{2}\right)=\mathfrak{M}_{2}, & L_{2}\left(\mathfrak{M}_{2}\right)=\mathcal{P}^{*} .
\end{array}
$$

By 1.8 the middle-spaces $\mathfrak{M}_{1}, \mathfrak{M}_{2}$ are homeomorphic. Hence, if $\mathfrak{M}_{1}$ is a dendrite, then $\mathfrak{M}_{2}$ is also a dendrite, and the theorem is obviously true in this case. So let us assume that $\mathfrak{M}_{1}$ is not a dendrite. Let $\Gamma_{1}^{1}, \cdots, \Gamma_{1}^{n}, \cdots$ be the sequence of proper cyclic elements of $\mathfrak{M}_{1}$, and let $h\left(\mathfrak{M}_{1}\right)=\mathfrak{M}_{2}$ be a topological mapping with the properties (i), (ii) described in 1.8. On setting $\Gamma_{2}^{n}=h\left(\Gamma_{1}^{n}\right)$, we obtain the sequence of the proper cyclic elements of $\mathfrak{M}_{2}$. Let $\mu_{i}^{n}\left(\mathfrak{M}_{i}\right)=\Gamma_{i}^{n}$, $i=1,2$, be the unique monotone retraction from $\mathfrak{M}_{i}$ onto $\Gamma_{i}^{n}$. By definition (cf. 1.9, 1.10)

$$
\begin{aligned}
& \Delta\left(T_{1}\right)=\left[L_{1} \mu_{1}^{1} M_{1}, \cdots, L_{1} \mu_{1}^{n} M_{1}, \cdots\right], \\
& \Delta\left(T_{2}\right)=\left[L_{2} \mu_{2}^{1} M_{2}, \cdots, L_{2} \mu_{2}^{n} M_{2}, \cdots\right] .
\end{aligned}
$$

Now clearly $h \mu_{1}^{n} h^{-1}$ is a monotone retraction from $\mathfrak{M}_{2}$ onto $\Gamma_{2}^{n}$. Since there exists just one such monotone retraction, it follows that $\mu_{2}^{n}=h \mu_{1}^{n} h^{-1}$. By 1.8 (ii), there follows the relation

$$
L_{1} \mu_{1}^{n} M_{1} \sim L_{2} \mu_{2}^{n} M_{2}(F), \quad n=1,2, \cdots,
$$

and the theorem is proved.

This argument shows that every homeomorphism $h\left(\mathfrak{M}_{1}\right)=\mathfrak{M}_{2}$ with the property 1.8 (ii) induces simultaneous arrangements of the elements of $\Delta\left(T_{1}\right)$, $\Delta\left(T_{2}\right)$ such that corresponding terms are $F$-equivalent. Simple examples show that no such statement holds in general for homeomorphisms $h\left(\mathfrak{M}_{1}\right)=\mathfrak{M}_{2}$ possessing only property 1.8 (i), even though $T_{1} \sim T_{2}(F)$. 


\section{MoNotone MAPPINGS OF 2-SPHERES AND 2-CELLS}

2.1. Let $M(P)=\mathfrak{M}$ be a continuous monotone mapping, where $\Phi$ is a 2 -sphere. Suppose that $\mathfrak{M}$ is cyclic (that is, $\mathfrak{M}$ has no cut points). Then $\mathfrak{M}$ is a 2-sphere (see Whyburn [6, p. 170, Theorem 2.1]).

2.2. Let $M(P)=\mathfrak{M}$ be a continuous monotone mapping, where $\Phi$ is a 2 -sphere. Then every proper cyclic element $\bar{C}$ of $\mathfrak{M}$ is a 2 -sphere. Indeed, there exists a (unique) monotone retraction $\mu(\mathfrak{R})=\bar{C}$ (cf. 1.2). Then $\mu M$ is a monotone mapping from $P$ onto $\bar{C}$. Since $\bar{C}$ is cyclic, it follows by 2.1 that $\bar{C}$ is a 2 -sphere.

2.3. Let $M(\mathscr{P})=\mathfrak{M}$ be a continuous monotone mapping, where $\mathcal{P}$ is a 2-cell, and $\mathfrak{M}$ is cyclic. Then $\mathfrak{M}$ is either a 2-cell or a 2-sphere. If $\mathfrak{M}$ is a 2-cell, then the boundary curve of $\mathscr{P}$ is mapped onto the boundary curve of $\mathfrak{M}$. If $\mathfrak{M}$ is a 2-sphere, then the boundary curve of $P$ is mapped onto a single point of $\mathfrak{M}$ (see Whyburn [6, p. 173, Theorem 2.41]).

2.4. Let $M(\mathscr{P})=\mathfrak{M}$ be a continuous monotone mapping, where $P$ is a 2 -cell. Then every proper cyclic element of $\mathfrak{M}$ is either a 2-cell or a 2-sphere. This follows from 2.3 by the argument used to derive 2.2 from 2.1 .

2.5. Let $M(\mathscr{P})=\mathfrak{M}$ be a continuous monotone mapping, where $\mathscr{P}$ is a 2-cell. Let $K$ denote the upper semi-continuous collection of maximal model continua determined by $M$ on $\mathbb{P}$ (cf. 1.3). Then $\mathfrak{M}$ is cyclic if and only if for every continuum $\gamma \in K$ the set $\Phi-\gamma$ is connected. Indeed, if $\gamma \in K$, then $M(\gamma)$ is a single point $\bar{x}$ of $\mathfrak{M}$, and $\mathscr{P}-\gamma=M^{-1}(\mathfrak{M}-\bar{x})$. Since $M$ is monotone, $M^{-1}(\mathfrak{M}-\bar{x})$ is connected if and only if $\mathfrak{M}-\bar{x}$ is connected, and the assertion follows.

Suppose now that $\mathcal{P}-\gamma$ is connected for every $\gamma \in K$. By the preceding remark, $\mathfrak{M}$ is then cyclic, and hence $\mathfrak{M}$ is a 2 -sphere or a 2 -cell by 2.3 . If $\mathfrak{M}$ is a 2 -sphere, then, by 2.3 , the boundary curve $C$ of $P$ is mapped onto a single point $\bar{y}_{0} \in \mathfrak{M}$, and hence $C$ is a subset of a continuum $\gamma_{0} \in K$, namely, of the continuum $\gamma_{0}=M^{-1}\left(\bar{y}_{0}\right)$. Conversely, if $C$ is a subset of some continuum $\gamma_{0} \in K$, then clearly $M(C)$ is a single point of $\mathfrak{M}$, and hence, in view of 2.3 , $\mathfrak{M}$ cannot be a 2-cell. Thus $\mathfrak{M}$ is a 2 -sphere, by 2.3 .

Summing up, if $P-\gamma$ is connected for every $\gamma \in K$, then $\mathfrak{M}$ is either a 2-cell or a 2-sphere. $\mathfrak{M}$ is a 2-cell if and only if the boundary curve $C$ of $\mathbb{P}$ is not a subset of any continuum $\gamma \in K$. $\mathfrak{M}$ is a 2-sphere if and only if $C$ is a subset of some continuum $\gamma_{0} \in K$.

2.6. Let $M(\mathscr{P})=\mathfrak{M}$ be a continuous monotone mapping, where $\mathscr{P}$ and $\mathfrak{M}$ are both 2-cells. By 2.3 the boundary curve $C$ of $\mathscr{P}$ is then mapped onto the boundary curve $\bar{C}$ of $\mathfrak{M}$. We assert that the mapping $M(C)=\bar{C}$ is monotone. Indeed, this mapping is non-alternating as a special case of Whyburn [6, p. 173, Theorem 2.52]), and hence it is monotone as a special case of Whyburn [6, p. 165, Theorem 1.2].

2.7. The following trivial remark is useful. Let $T(\mathscr{P})=\mathbb{P}^{*}$ be a continuous 
mapping from a Peano space $\mathcal{P}$ onto a Peano space $\mathbb{P}^{*}$, and let $F$ be a continuum in $P$. Then $T$ is monotone on $F$ if and only if the set $F T^{-1}\left(p^{*}\right)$ is connected (possibly empty) for every choice of the point $p^{*} \in \mathcal{P}^{*}$. Indeed, if $T(F)=F^{*}$, and if we denote, for clarity, by $T_{F}$ the mapping $T$ thought of as operating from $F$ only, then clearly $T_{F}^{-1}\left(p^{*}\right)=F T^{-1}\left(p^{*}\right)$ for every point $p^{*} \in P^{*}$, and the assertion follows.

2.8. Let $M(S)=\vec{S}$ be a continuous monotone mapping from a 2 -sphere $S$ onto a 2 -sphere $\bar{S}$, and let $\bar{A}$ be a 2-cell on $\bar{S}$. According to Youngs (see [7, p. 759]), we have then the following theorem.

Modification Theorem of Youngs. Under the conditions just stated, there exists a continuous monotone mapping $M_{1}(S)=\bar{S}$ with the following properties.

(i) $M_{1}(x)=M(x)$ for $x \notin M^{-1}\left(\bar{A}^{0}\right)$, where $\bar{A}^{0}$ is the interior of the 2-cell $\bar{A}$.

(ii) $M_{1}$ maps the set $M^{-1}\left(\bar{A}^{0}\right)$ topologically onto $\bar{A}^{0}$.

Let us note the following immediate implications of (i) and (ii).

(iii) If $\bar{x}$ is a point in $\bar{A}^{0}$, then $M_{1}^{-1}(\bar{x})$ is a single point in $M^{-1}\left(\bar{A}^{0}\right)$.

(iv) If $\bar{x} \notin \bar{A}^{0}$, then $M_{1}^{-1}(\bar{x})=M^{-1}(\bar{x})$.

(v) If $F$ is any set in $S$, then $\left(\bar{S}-\bar{A}^{0}\right) M_{1}(F)=\left(\bar{S}-\bar{A}^{0}\right) M(F)$.

(vi) If $M_{1}(x) \neq M(x)$, then $\rho\left[M_{1}(x), M(x)\right] \leqq d(\bar{A})$ (cf. (i), (ii)).

(vii) $d\left[M_{1}^{-1}(\bar{x})\right] \leqq d\left[M^{-1}(\bar{x})\right]$ for every point $\bar{x} \in \bar{S}$ (cf. (iii), (iv)).

(viii) If $G$ is a continuum such that $G \subset S-M^{-1}\left(\bar{A}^{0}\right)$ and $M$ is monotone on $G$, then $M_{1}$ is also monotone on $G$ (cf. (iv) and 2.7).

2.9. In applying the modification theorem of 2.8 , it will be convenient to use the following special assumptions and notations. We shall assume that $S$ coincides with the unit sphere $x^{2}+y^{2}+z^{2}=1$ in Euclidean $x y z$ space and $\bar{S}$ coincides with the unit sphere $\bar{x}^{2}+\bar{y}^{2}+\bar{z}^{2}=1$ in Euclidean $\bar{x} \bar{y} \bar{z}$ space. $E$ will denote the equator of $S$, that is, the great circle $z=0$. The points $(0,0,1)$, $(0,0,-1)$ will be termed the north pole $\nu$ and the south pole $\sigma$ of $S$ 'respectively. $S_{\sigma}, S_{\sigma}^{0}$ will denote the closed and open southern hemispheres on $S$, that is, the subsets of $S$ characterized by the inequalities $z \leqq 0, z<0$. The closed and open northern hemispheres $S_{\nu}, S_{\nu}^{0}$ are defined similarly. Finally, $\bar{S}_{\sigma}, \cdots, \bar{S}_{\nu}^{0}, \bar{E}$ will have analogous meanings relative to the sphere $\bar{S}$.

Let $\bar{A}$ be a 2-cell on $\bar{S}$, and let $\bar{A}^{0}$ denote the interior of $\bar{A}$. The 2-cell $\bar{A}$ will be termed admissible if either $\bar{A}^{0} \bar{E}=0$, or if $\bar{A}^{0} \bar{E} \neq 0$ and the boundary of $\bar{A}$ intersects the equator $\bar{E}$ in exactly two points. A finite system of 2-cells $\bar{A}_{1}, \cdots, \bar{A}_{n}$ on $\bar{S}$ will be termed admissible if (1) $\bar{A}_{i}^{0} \bar{A}_{j}^{0}=0$ for $i \neq j$, and (2) each $\bar{A}_{i}, i=1, \cdots, n$, is admissible.

2.10. Using the terminology of 2.9 , we have the following lemma.

Let $\eta>0$ be given. Then there exist three admissible systems $\bar{A}_{1}, \cdots, \bar{A}_{a}$; $\bar{B}_{1}, \cdots, \bar{B}_{b} ; \bar{C}_{1}, \cdots, \bar{C}_{c}$ of 2-cells on $\bar{S}$, such that (1) each one of the 2-cells involved has a diameter less than $\eta$, and (2) $\bar{S}=\bar{A}_{1}^{0}+\cdots+\bar{C}_{c}^{0}$.

The construction of these systems is entirely analogous to that used by 
Youngs (see [7, pp. 760-761]); the additional condition of admissibility, not needed by Youngs, obviously implies no difficulty in applying his construction.

2.11. Let $M(S)=\bar{S}$ be a continuous monotone mapping from the unit sphere $S$ onto the unit sphere $\bar{S}$, such that $M(E)=\bar{E}$ and $M$ is monotone on $E$ (see 2.9). Let $\bar{A}$ be an admissible 2-cell on $\bar{S}$ (see 2.9). Then there exists a continuous monotone mapping $\bar{M}(S)=\bar{S}$ with the following properties.

(1) $\bar{M}(E)=\bar{E}$, and $\bar{M}$ is monotone on $E$.

(2) $d\left[\bar{M}^{-1}(\bar{x})\right] \leqq d\left[M^{-1}(\bar{x})\right]$ for every point $\bar{x} \in \bar{S}$.

(3) If $\bar{x} \in \bar{A}^{0}$, then $\bar{M}^{-1}(\bar{x})$ is a single point in $M^{-1}\left(\bar{A}^{0}\right)$.

(4) If $x \in M^{-1}\left(\bar{A}^{0}\right)$, then $\bar{M}(x)=M(x)$.

(5) If $x \in M^{-1}\left(\bar{A}^{0}\right)$, then $\bar{M}(x) \in \bar{A}^{0}$.

Proof. Let $M_{1}(S)=\bar{S}$ be a continuous monotone mapping, obtained by the modification theorem of Youngs; then $M_{1}$ possesses the properties (i)(viii) listed in 2.8. Noting that $\bar{A}$ is admissible, we distinguish between the following two cases.

Case I. $\bar{A}^{0} \bar{E}=0$. Then the mapping $\bar{M} \equiv M_{1}$ obviously satisfies our requirements.

Case II. $\bar{A}^{0} \bar{E} \neq 0$. Then the boundary curve of $\bar{A}$ intersects $\bar{E}$ in exactly two points, since $\bar{A}$ is admissible. Let $\bar{\phi}, \bar{q}$ be these points of intersection, and let $\bar{\alpha}, \bar{\beta}$ be the two sub-arcs of $\bar{E}$ with end points $\bar{p}, \bar{q}$, where $\bar{\alpha} \subset \bar{A}$. Let us denote by $\bar{B}$ the 2 -cell $\bar{S}-\bar{A}^{0}$. Then we have the relations

$$
\bar{\alpha} \subset \bar{A}, \bar{\beta} \subset \bar{B}, \bar{\alpha} \bar{B}=\bar{\beta} \bar{A}=\bar{p}+\bar{q} \text {. }
$$

We proceed to verify the following facts.

(a) $M_{1}$ is monotone on $E$.

(b) $M_{1}(E)=\bar{E}_{1}$ is a simple closed curve.

(c) $\bar{E}_{1}=\bar{\beta}+\bar{\alpha}_{1}$, where $\bar{\alpha}_{1}$ is a simple arc with end points $\bar{p}, \bar{q}$, and $\bar{\alpha}_{1} \bar{B}=\bar{p}+\bar{q}$. In other words, $\bar{\alpha}_{1}$ is contained in $\bar{A}^{0}$ except for its end points $\bar{\phi}, \bar{q}$.

Proof of (a). Let $\bar{x}$ be any point in $\bar{S}$. If $\bar{x} \in \bar{A}^{0}$, then $M_{1}^{-1}(\bar{x})$ is a single point by 2.8 (iii), and hence $E M_{1}^{-1}(\bar{x})$ is either a single point or empty. If $\bar{x} \notin \bar{A}^{0}$, then $E M_{1}^{-1}(\bar{x})=E M^{-1}(\bar{x})$ by 2.8 (iv). Hence $E M_{1}^{-1}(\bar{x})$ is connected, since $E M^{-1}(\bar{x})$ is connected as a consequence of the assumption that $M$ is monotone on $E$. Thus $E M_{1}^{-1}(\bar{x})$ is connected (possibly empty) for every point $\bar{x} \in \bar{S}$, and hence $M_{1}$ is monotone on $E$ (see 2.7).

Proof of (b). Note first that $M_{1}(E) \bar{B}=M(E) \bar{B}=\bar{E} \bar{B}=\bar{\beta}$ (cf. 2.8 (v)). Thus $\bar{\beta} \subset M_{1}(E)$, and hence $M_{1}(E)$ does not reduce to a single point. Since $M_{1}$ is monotone on $E$ and $E$ is a simple closed curve, it follows that $M_{1}(E)=\bar{E}_{1}$ is also a (nondegenerate) simple closed curve (see Whyburn [6, p. 165, Theorem $1.1])$.

Proof of (c). We already proved that $\bar{E}_{1}$ is a simple closed curve that contains the simple arc $\bar{\beta}$. Hence $\bar{E}_{1}=\bar{\beta}+\bar{\alpha}_{1}$, where $\bar{\alpha}_{1}$ is a simple arc with end points $\bar{\phi}, \bar{q}$, and $\bar{\alpha}_{1} \bar{\beta}=\bar{\phi}+\bar{q}$. There remains to show that $\bar{\alpha}_{1} \bar{B}=\bar{\phi}+\bar{q}$. Now we 
noted, in proving (b), that $\bar{E}_{1} \bar{B}=M_{1}(E) \bar{B}=\bar{\beta}$. Since $\bar{\alpha}_{1} \subset \bar{E}_{1}$, it follows that $\bar{\alpha}_{1} \bar{B}=\bar{\alpha}_{1} \bar{E}_{1} \bar{B}=\bar{\alpha}_{1} \bar{\beta}=\bar{p}+\bar{q}$.

Now that (a), (b), (c) are verified, let us consider the 2-cell $\bar{A}$. The points $\bar{p}, \bar{q}$ lie on the boundary curve of $\bar{A}$, and are joined by two simple arcs $\bar{\alpha}, \bar{\alpha}_{1}$ that are contained in $\bar{A}^{0}$ except for their common end points $\bar{p}, \bar{q}$. There follows the existence of a topological mapping $\bar{h}(\bar{A})=\bar{A}$ that maps $\bar{\alpha}_{1}$ onto $\bar{\alpha}$ and reduces to the identity on the boundary curve of $\bar{A}$ (cf. Newman [8, pp. 165-169]). Then the mapping $\bar{H}(\bar{S})=\bar{S}$ defined by

$$
\bar{H}(\bar{x})=\begin{aligned}
& \bar{x} \text { for } \bar{x} \notin \bar{A}^{0}, \\
& \bar{h}(\bar{x}) \text { for } \bar{x} \in \bar{A}^{0}
\end{aligned}
$$

is a topological mapping from $\bar{S}$ onto itself. Let us define now a mapping $\bar{M}(S)=\bar{S}$ as follows:

$$
\bar{M}(x)=\bar{H} M_{1}(x),
$$

$x \in S$.

In view of (a), (b), (c), the mapping $\bar{M}$ obviously satisfies all of our requirements.

2.12. Let $M(S)=\bar{S}$ be a continuous monotone mapping from the unit sphere $S$ onto the unit sphere $\bar{S}$, such that $M(E)=\bar{E}$ and $M$ is monotone on $E$. Let $\bar{A}_{1}, \cdots, \bar{A}_{n}$ be an admissible system of 2-cells on $\bar{S}$ (cf. 2.9). Then there exists a continuous monotone mapping $M_{*}(S)=\bar{S}$ with the following properties.

(1) $M_{*}(E)=\bar{E}$, and $M_{*}$ is monotone on $E$.

(2) $d\left[M_{*}^{-1}(\bar{x})\right] \leqq d\left[M^{-1}(\bar{x})\right]$ for every point $\bar{x} \in \bar{S}$.

(3) If $\bar{x} \in \bar{A}_{1}^{0}+\cdots+\bar{A}_{n}^{0}$, then $M_{*}^{-1}(\bar{x})$ is a single point.

(4) $\rho\left[M(x), M_{*}(x)\right] \leqq \max \left[d\left(\bar{A}_{1}\right), \cdots, d\left(\bar{A}_{n}\right)\right]$ for every point $x \in S$.

Proof. Apply the result of 2.11 to $M$, using the 2-cell $\bar{A}_{1}$, obtaining a mapping $\bar{M}_{1}$. Apply the same process to $\bar{M}_{1}$, using the 2-cell $\bar{A}_{2}$, obtaining a mapping $\bar{M}_{2}$, and so on. There results a sequence of mappings $\bar{M}_{1}, \bar{M}_{2}, \cdots, \bar{M}_{n}$. We assert that the mapping $M_{*} \equiv M_{n}$ satisfies our requirements. Indeed, (1), (2), (3) are immediate consequences of 2.11 (1), 2.11 (2), 2.11 (3). As regards (4), observe that the sets $M^{-1}\left(\bar{A}_{1}^{0}\right), \cdots, M^{-1}\left(\bar{A}_{n}^{0}\right)$ are disjoint, and hence a point $x \in S$ lies in at most one of these sets. If $x$ does not lie in the sum of these sets, then $M_{*}(x)=M(x)$ in view of 2.11 (4). If $x \in M^{-1}\left(\bar{A}_{i}^{0}\right)$ for a certain $i$, then clearly $M_{*}(x)=\bar{M}_{i}(x)$, and hence in view of $2.11(5), M_{*}(x)$ and $M(x)$ both lie in $\bar{A}_{i}^{0}$. Thus (4) follows.

2.13. Approximation Theorem. Let $M(S)=\bar{S}$ be a continuous monotone mapping from the unit sphere $S$ onto the unit sphere $\bar{S}$, such that $M(E)=\bar{E}$ and $M$ is monotone on $E$ (cf. 2.9). Let $\epsilon>0$ be given. Then there exists a topological mapping $H(S)=\bar{S}$, such that $H(E)=\bar{E}$, and $\rho[M(x), H(x)]<\epsilon$ for every point $x \in S$.

Proof. Let $\eta=\epsilon / 3$. Choose three admissible systems $\bar{A}_{1}, \cdots, \bar{A}_{a}$; 
$\bar{B}_{1}, \cdots, \bar{B}_{b} ; \bar{C}_{1}, \cdots, \bar{C}_{c}$ of 2 -cells on $\bar{S}$ according to 2.10 . Apply the result of 2.12 - to $M$ and the system $\bar{A}_{1}, \cdots, \bar{A}_{a}$, obtaining a mapping $M_{* 1}$. Apply the same process to $M_{* 1}$ and $\bar{B}_{1}, \cdots, \bar{B}_{b}$, obtaining a mapping $M_{* 2}$. Apply the same process to $M_{* 2}$ and $\bar{C}_{1}, \cdots, \bar{C}_{c}$, obtaining a mapping $M_{* 3}$. We assert that $H \equiv M_{* 3}$ satisfies our requirements. In view of 2.12 (1), 2.12 (4), clearly $H(E)=\bar{E}$ and $\rho[M(x), H(x)]<3 \eta=\epsilon$ for every $x \in S$. There remains to show that $H$ is bi-unique. Now let $\bar{x}$ be any point of $\bar{S}$. Since $\bar{A}_{1}^{0}+\cdots+\bar{C}_{c}=\bar{S}$, it follows by 2.12 (3) that one at least of the sets $M_{* 1}^{-1}(\bar{x}), M_{* 2}^{-1}(\bar{x}), M_{* 3}^{-1}(\bar{x})$ reduces to a single point. Since $d\left[M_{* 3}^{-1}(\bar{x})\right]$ $\leqq d\left[M_{* 2}^{-1}(\bar{x})\right] \leqq d\left[M_{* 1}^{-1}(\bar{x})\right]$ (cf. $2.12(2)$ ), it follows that $H^{-1}(\bar{x})=M_{* 3}^{-1}(\bar{x})$ reduces to a single point for every $\bar{x} \in \bar{S}$. Since $H(S)=\bar{S}$ is continuous and biunique and $S, \bar{S}$ are compact spaces, it follows that $H$ is topological.

2.14. For comparison and reference, we quote two important approximation theorems due to Youngs ([7, p. 760, Theorem 11.1, and p. 76i, Theorem 11.3]).

Approximation Theorem (Youngs). Let $M(S)=\bar{S}$ be a continuous monotone mapping from a 2-sphere $S$ onto a 2-sphere $\bar{S}$. Let $\epsilon>0$ be given. Then there exists a topological mapping $H(S)=\bar{S}$, such that $\rho[M(x), H(x)]<\epsilon$ for every point $x \in S$.

Approximation Theorem (Youngs). Let $M(S)=\bar{S}$ be a continuous monotone mapping from a 2-sphere $S$ onto a 2-sphere $\bar{S}$. Let $p_{1}, \cdots, p_{n}$ be a finite system of points on $\bar{S}$. Let $\epsilon>0$ be given. Then there exists a continuous monotone mapping $M_{*}(S)=\bar{S}$ with the following properties: (i) $M_{*}(x)=M(x)$ for $x \in M^{-1}\left(p_{1}+\cdots+p_{n}\right)$. (ii) $M_{*}$ maps the set $S-M^{-1}\left(p_{1}+\cdots+p_{n}\right)$ topologically upon the set $\bar{S}-\left(\bar{p}_{1}+\cdots+\bar{p}_{n}\right)$. (iii) $\rho\left[M(x), M_{*}(x)\right]<\epsilon$ for every $x \in S$.

Our proof of the theorem of 2.13 is entirely analogous to the proof of Youngs for the approximation theorems just quoted, except for the complications implied by our additional conditions regarding the equators $E, \bar{E}$. Conversely, the reader will have little difficulty in reconstructing the proofs of Youngs on the basis of the argument used to establish the theorem of 2.13.

The approximation theorems of 2.13 and 2.14 deal with mappings from 2 -spheres. We proceed to derive from these results analogous approximation theorems for mappings from 2-cells.

2.15. ApProximation TheOREM. Let $M(P)=\mathfrak{M}$ be a continuous monotone mapping, where $\mathcal{P}$ is a 2-cell and $\mathfrak{M}$ is a 2-sphere; as noted in 2.3; the boundary curve of $\Phi$ is then mapped onto a single point $\bar{y}_{0}$ of $\mathfrak{M}$. Let $\epsilon>0$ be given. Then there exists a continuous monotone mapping $M_{*}(\mathscr{P})=\mathfrak{M}$ with the following properties: (i) $M_{*}$ maps the boundary curve of $\mathcal{P}$ onto the single point $\bar{y}_{0}$. (ii) $M_{*}$ maps the set $\mathbb{P}-M^{-1}\left(\bar{y}_{0}\right)$ topologically onto the set $\mathfrak{M}-\bar{y}_{0}$. (iii) $\rho\left[M_{*}(x), M(x)\right]<\epsilon$ for every point $x \in$

Proof. Without loss of generality, we can assume that $P$ coincides with 
the southern hemisphere $S_{\sigma}$ on the unit sphere $S$, while $\mathfrak{M}$ coincides with the unit sphere $\bar{S}$ (cf. 2.9). Let us extend the definition of the mapping $M\left(S_{\sigma}\right)=\bar{S}$ by setting $M(x)=\bar{y}_{0}$ for $x \in S_{\nu}^{0}$. Then the extended mapping $M(S)=\bar{S}$ is clearly continuous and monotone, and $M^{-1}(\bar{x})$ is unchanged if $\bar{x} \neq \bar{y}_{0}$, while $M^{-1}\left(\bar{y}_{0}\right)$ is augmented by $S_{\nu}^{0}$. The second approximation theorem of 2.14 , applied with $n=1, \bar{p}_{1}=\bar{y}_{0}$ yields a mapping $M_{*}$ which clearly satisfies our requirements.

2.16. We shall need the following extension theorem (cf. 2.18). Using the notations of 2.9 , let $M\left(S_{\sigma}\right)=\bar{S}_{\sigma}$ be a continuous monotone mapping. Then there exists a continuous monotone mapping $M_{*}(S)=\bar{S}$, such that $M_{*}(x)$ $=M(x)$ for $x \in S_{\text {. }}$.

Proof. By 2.6, $M(E)=\bar{E}$ and $M$ is monotone on $E$. Hence, as a special case of Whyburn $[6$, p. 180, Theorem 4.5], there exists a continuous monotone mapping $M_{\nu}\left(S_{v}\right)=\bar{S}_{v}$, such that $M_{\nu}(x)=M(x)$ for $x \in E$. Let us define a mapping $M_{*}(S)=\bar{S}$ as follows:

$$
M_{*}(x)=\left\{\begin{array}{lll}
M_{\nu}(x) & \text { if } & x \in S_{\nu} \\
M(x) & \text { if } & x \in S_{\sigma} .
\end{array}\right.
$$

Clearly, $M_{*}$ is single-valued and continuous. There remains to show that $M_{*}$ is monotone. Now if $\bar{x}$ is any point of $\bar{S}$, then clearly

$$
M_{*}^{-1}(\bar{x})=\left\{\begin{array}{l}
M^{-1}(\bar{x}) \text { if } \quad \bar{x} \in \bar{S}_{\sigma}, \\
M_{\nu}^{-1}(\bar{x}) \text { if } \bar{x} \in \bar{S}_{\nu}^{0}, \\
M^{-1}(\bar{x})+M_{\nu}^{-1}(\bar{x}) \text { if } \quad \bar{x} \in \bar{E} .
\end{array}\right.
$$

Since $M$ is monotone on $S_{\sigma}$ and $M_{\nu}$ is monotone on $S_{\nu}$, the sets $M^{-1}(\bar{x})$, $M_{\nu}^{-1}(\bar{x})$ are connected (possibly empty), and hence $M_{*}^{-1}(\bar{x})$ is connected if $\bar{x} \notin \bar{E}$. If $\bar{x} \in \bar{E}$, then the relations $M(E)=\bar{E}, M_{\nu}(x)=M(x)$ for $x \in E$ imply that the sets $M^{-1}(\bar{x}), M_{\nu}^{-1}(\bar{x})$ have a non-empty intersection, and hence their sum $M_{*}^{-1}(\bar{x})$ is connected. Thus $M_{*}$ is monotone.

2.17. Approximation Theorem (cf. 2.18). Let $M(\mathcal{P})=\mathbb{P}^{*}$ be a continuous monotone mapping, where $\mathcal{P}$ and $\mathcal{P}^{*}$ are both 2-cells. Let $\epsilon>0$ be given. Then there exists a topological mapping $H(\mathscr{P})=\mathcal{P}^{*}$, such that $\rho[M(x), H(x)]<\epsilon$ for every point $x \in \mathcal{P}$.

Proof. Using the terminology of 2.9, we can assume without loss of generality that $\mathcal{P}, \mathbb{P}^{*}$ coincide with the hemispheres $S_{\sigma}, \bar{S}_{\sigma}$ on the unit spheres $S, \bar{S}$ respectively. Obviously, we can also assume that $0<\epsilon<1$. By 2.16 we have then a continuous monotone mapping $M_{*}(S)=\bar{S}$ such that $M_{*}(x)=M(x)$ for $x \in S_{\sigma}$. By 2.6, $M(E)=\bar{E}$ and $M$ is monotone on $E$. Since $M_{*} \equiv M$ on $E$, it follows that $M_{*}(E)=\bar{E}$ and $M_{*}$ is monotone on $E$. By 2.13, applied to $M_{*}$, there follows the existence of a topological mapping $H(S)=\bar{S}$, such that 
$H(E)=\bar{E}$ and $\rho\left[M_{*}(x), H(x)\right]<\epsilon$ for every point $x \in S$. In particular, since $M_{*} \equiv M$ on $S_{\sigma}$, it follows that $\rho[M(x), H(x)]<\epsilon$ for every point $x \in S_{\sigma}$. There remains to show that $H\left(S_{\sigma}\right)=\bar{S}_{\sigma}$. Now since $H(S)=\bar{S}$ is topological and $H(E)=\bar{E}$, we have either $H\left(S_{\sigma}\right)=\bar{S}_{\sigma}$ or $H\left(S_{\sigma}\right)=\bar{S}_{\nu}$. Suppose that $H\left(S_{\sigma}\right)=\bar{S}_{\nu}$. Let $\bar{\nu}$ denote the north pole of $\bar{S}$. Then $H^{-1}(\bar{\nu})=x_{\nu}$ is a point in $S_{\sigma}$, and hence $M\left(x_{\nu}\right)$ is a point in $\bar{S}_{\sigma}$. Hence we should have the inequalities

$$
\pi / 2 \leqq \rho\left[M\left(x_{v}\right), H\left(x_{v}\right)\right]<\epsilon<1 .
$$

Thus the assumption $H\left(S_{\sigma}\right)=\bar{S}_{\nu}$ leads to a contradiction, and hence $H\left(S_{\sigma}\right)=\bar{S}_{\sigma}$.

2.18. Let us add some remarks concerning previous literature. The extension theorem of 2.16 has already been stated by Morrey [3], in an obviously equivalent form. In fact, Morrey attempted to prove a more general extension theorem (see [3, p. 37, Lemma 2]), which unfortunately seems to be false. Let $D$ denote the unit circular disc $u^{2}+v^{2} \leqq 1$ in a Euclidean $u v$ plane. Let $P$ be the perimeter of $D$, and let $D_{l}, D_{r}$ be the subsets of $D$ characterized by the inequalities $u \leqq 0, u \geqq 0$ respectively. Finally, let $P_{l}, P_{r}$ be the subsets of $P$ characterized by the inequalities $u \leqq 0, u \geqq 0$. Consider now, in a Euclidean $\bar{u} \bar{v}$ plane, the point set $\bar{D}$ defined as the sum of the two circular discs $\bar{D}_{l}:(\bar{u}+1)^{2}+\bar{v}^{2} \leqq 1, \bar{D}_{r}:(\bar{u}-1)^{2}+\bar{v}^{2} \leqq 1$, and let $\bar{P}_{l}, \bar{P}_{r}$ denote the perimeters of $\bar{D}_{l}, \bar{D}_{r}$ respectively. Clearly, we have then a continuous monotone mapping $M\left(D_{l}\right)=\bar{D}_{l}$ which is topological except for the fact that the vertical diameter of $D$ is mapped onto the single point $(0,0)$. Furthermore, we can require that while the point $(u, v)$ describes $P_{l}$ in the counterclockwise sense, its image $(\bar{u}, \bar{v})$ describes $\bar{P}_{l}$ in the counterclockwise sense. Similarly, we have a continuous monotone mapping $M_{r}\left(D_{r}\right)=\bar{D}_{r}$ with analogous properties with respect to $D_{r}$, with this difference: we require now that while $(u, v)$ describes $P_{r}$ in the counterclockwise sense, its image describes $\bar{P}_{r}$ in the clockwise sense. Let us now define the mapping $M(D)=\bar{D}$ as follows: $M \equiv M_{l}$ on $D_{l}$, and $M \equiv M_{r}$ on $D_{r}$. Clearly, $M$ is single-valued, continuous, and monotone on $D$, and seems to satisfy all the requirements of the theorem of Morrey referred to above. Suppose now that we have, as asserted by Morrey, an extension of $M$ to the whole (finite) $u v$ plane such that the extended transformation $M$ is topological for $u^{2}+v^{2}>1$. If $C$ is a simple closed curve in the $u v$ plane, then let $i(\bar{u}, \bar{v}, C)$ denote the topological index of the point $(\bar{u}, \bar{v})$ with respect to the image of $C$ under $M$; where $C$ is oriented in the counterclockwise sense. Then clearly $i(1,0, P)=-1, i(-1,0, P)=+1$. Now let us choose $C$ as a circle with center $(0,0)$ and a radius slightly exceeding unity. Then we have, on account of the continuity of $M, i(1,0, C)=i(1,0, P)=-1, i(-1,0, C)$ $=i(-1,0, P)=+1$. On the other hand, since $M$ is topological for $u^{2}+v^{2}>1$, $M(C)$ is a simple closed curve that contains the points $(1,0)$ and $(-1,0)$ of the $\bar{u} \bar{v}$ plane in its interior, and hence we must have $i(1,0, C)=i(-1,0, C)$. This contradiction shows that there exists no extension of $M$ with the asserted properties. 
The preceding counter-example is quite similar to and has been suggested by a counter-example devised by Youngs for an analogous purpose (cf. Youngs [7, pp. 765-766]).

As regards the approximation theorem of 2.17 , this theorem also occurs in Morrey's paper [3]. In fact, the argument used by Morrey is brief and elementary, while our proof, even though reasonably compact, uses advanced tools (for example, the topological characterization of the 2-sphere). However, the writer agrees with the opinion expressed by Youngs that the theorem is perhaps deeper than the elementary comments of Morrey might lead one to believe (cf. Youngs [7, p. 761]).

If $M(P)=\Phi^{*}$ is a monotone continuous mapping and $\mathcal{P}, \mathbb{P}^{*}$ are closed linear intervals, then it is quite obvious that $M$ is the uniform limit of homeomorphisms. The approximation theorems of $2.13,2.14,2.17$ are plausible generalizations of this trivial fact.

\section{F-equivalent MAPPINGS OF 2-CELls}

3.1. We shall study presently a pair of continuous mappings $T_{1}\left(\mathscr{P}_{1}\right)=\Phi^{*}$, $T_{2}\left(\mathscr{P}_{2}\right)=\mathrm{P}^{*}$, where $\mathcal{P}_{1}$ and $\mathcal{P}_{2}$ are both 2 -cells.

3.2. Given $T_{1}, T_{2}$ as in 3.1 , let us suppose that $T_{1} \sim T_{2}(F)$. We assert that $T_{1}, T_{2}$ admit of simultaneous factorizations of the form

$$
\begin{array}{lll}
T_{1}=L M_{1}, & M_{1}\left(\mathbb{P}_{1}\right)=\mathfrak{M}, & L(\mathfrak{M})=\mathbb{P}^{*}, \\
T_{2}=L M_{2}, & M_{2}\left(\mathbb{P}_{2}\right)=\mathfrak{M}, & L(\mathfrak{M})=\mathbb{P}^{*} .
\end{array}
$$

In other words, we can arrange for factorizations for $T_{1}, T_{2}$ with the same middle-space $\mathfrak{M}$ and the same light factor $L$.

Proof. Let us start with any monotone-light factorizations

$$
\begin{aligned}
& T_{1}=\bar{L}_{1} \bar{M}_{1}, \quad \bar{M}_{1}\left(\mathscr{P}_{1}\right)=\mathfrak{M}_{1}, \quad \bar{L}_{1}\left(\mathfrak{M}_{1}\right)=\mathbb{P}^{*}, \\
& T_{2}=\bar{L}_{2} \bar{M}_{2}, \quad \bar{M}_{2}\left(\mathscr{P}_{2}\right)=\mathfrak{M}_{2}, \quad \bar{L}_{2}\left(\mathfrak{M}_{2}\right)=\mathscr{P}^{*}
\end{aligned}
$$

for $T_{1}, T_{2}$. By 1.8 there exists then a topological mapping $h\left(\mathfrak{M}_{1}\right)=\mathfrak{M}_{2}$ such that $\bar{L}_{1}\left(\bar{x}_{1}\right)=\bar{L}_{2} h\left(\bar{x}_{1}\right)$ for every point $\bar{x}_{1} \in \mathfrak{M}_{1}$. Let us put $M_{1}=h \bar{M}_{1}$. Then $M_{1}$ is a monotone mapping from $\mathbb{P}_{1}$ onto $\mathfrak{M}_{2}$, and we have

$$
T_{1}=\bar{L}_{1} \bar{M}_{1}=\bar{L}_{2} h \bar{M}_{1}=\bar{L}_{2} M_{1}, \quad M_{1}\left(\mathscr{P}_{1}\right)=\mathfrak{M}_{2}, \quad \bar{L}_{2}\left(\mathfrak{M}_{2}\right)=\mathbb{P}^{*} .
$$

Hence, if we put $L=\bar{L}_{2}, \mathfrak{M}=\mathfrak{M}_{2}, M_{1}=h \bar{M}_{1}, M_{2}=\bar{M}_{2}$, then the formulas (1), (2) hold.

3.3. Suppose conversely that $T_{1}, T_{2}$, given as in 3.1 , admit of simultaneous factorizations of the form

$$
\begin{array}{lll}
T_{1}=L M_{1}, & M_{1}\left(\mathscr{P}_{1}\right)=\mathfrak{M}, & L(\mathfrak{M})=\mathbb{P}^{*}, \\
T_{2}=L M_{2}, & M_{2}\left(\mathcal{P}_{2}\right)=\mathfrak{M}, & L(\mathfrak{M})=\mathcal{P}^{*} .
\end{array}
$$

For brevity, we shall then say that $T_{1}, T_{2}$ satisfy the hypothesis (K). Morrey 
[3] attempted to prove the theorem that the hypothesis $(\mathrm{K})$ implies the relation $T_{1} \sim T_{2}(F)$. Youngs discovered the regrettable fact that this theorem is false (see Youngs [7]). There arises thus the fundamental question: under what additional assumptions will the relation $T_{1} \sim T_{2}(F)$ follow? As mentioned in the introduction, a detailed study of this question is contained in an important paper of Youngs for the case when the initial spaces $P_{1}, P_{2}$ are 2 -spheres. Youngs also studied the case when $\mathcal{P}_{1}, \boldsymbol{P}_{2}$ are 2-cells, but his researches were interrupted by an assignment to war work in England. In view of the important role played by the relation $T_{1} \sim T_{2}(F)$ in certain applications, the failure of the theorem of Morrey creates a regrettable but not altogether hopeless situation. Indeed, a study of the applications reveals the fact that certain very special cases of that theorem would be adequate to meet present needs. We proceed to discuss these special cases.

3.4. In view of the applications, the validity of the following special statements is a matter of considerable interest.

Statement (a). If $T_{1}, T_{2}$ satisfy the hypothesis (K), and if the middlespace $\mathfrak{M}$ is a simple arc (cf. 3.3), then $T_{1} \sim T_{2}(F)$.

Statement (b). If $T_{1}, T_{2}$ satisfy the hypothesis (K), and if the middlespace $\mathfrak{M}$ is a 2-cell (cf. 3.3), then $T_{1} \sim T_{2}(F)$.

Statement (c). If $T_{1}, T_{2}$ satisfy the hypothesis $(\mathrm{K})$, and if the middlespace $\mathfrak{M}$ is a 2 -sphere (cf. 3.3), then $T_{1} \sim T_{2}(F)$.

Of course, if the general theorem asserted by Morrey were true, then these statements were also true. We shall now see that (a) and (c) are false. This negative result will then be supplemented by a discussion of two positive results. We shall verify that (b) is true, and that (c) can be turned into a valid statement by the addition of a further condition.

3.5. Let $u, v$ be Cartesian coordinates in a $u v$ plane, let $\alpha, \beta$ be Cartesian coordinates in an $\alpha \beta$ plane, and finally let $\xi, \eta, \zeta$ be Cartesian coordinates in a $\xi \eta \zeta$ space. Let us choose $\mathcal{P}_{1}, \mathcal{P}_{2}, \mathrm{P}^{*}, T_{\mathrm{m}}, T_{2}$ as follows:

$$
\begin{array}{ll}
\mathcal{P}_{1}: & u^{2}+v^{2} \leqq 1 . \quad \mathbb{P}_{2}: \quad 0 \leqq \alpha \leqq 1,0 \leqq \beta \leqq 1 \\
\mathbb{P}^{*}: & 0 \leqq \xi \leqq 1, \eta=0, \zeta=0 . \\
T_{1}: & \xi=\left(u^{2}+v^{2}\right)^{1 / 2}, \eta=0, \zeta=0,(u, v) \in \mathbb{P}_{1} \\
T_{2}: & \xi=\alpha, \eta=0, \zeta=0,(\alpha, \beta) \in \mathcal{P}_{2} .
\end{array}
$$

Obviously, $T_{1}, T_{2}$ are both monotone. Thus on setting $M_{1}=T_{1}, M_{2}=T_{2}$, $\mathfrak{M}=\mathbb{P}^{*}$ and choosing $L$ as the identity, it is clear that $T_{1}, T_{2}$ satisfy the assumptions of statement (a) in 3.4. On the other hand, the relation $T_{1} \sim T_{2}(F)$ fails to hold. Indeed, let $H$ be any topological mapping from $\mathcal{P}_{1}$ onto $\boldsymbol{P}_{2}$. Then $H$ maps the perimeter of $P_{1}$ onto the perimeter of $P_{2}$. Hence we have a point $\left(u_{0}, v_{0}\right)$ on the perimeter of $\mathcal{P}_{1}$ which is carried by $H$ into the point $(0,0)$ on the perimeter of $\mathcal{P}_{2}$. Then clearly $\rho\left[T_{1}\left(u_{0}, v_{0}\right), T_{2} H\left(u_{0}, v_{0}\right)\right]=1$. In other words, $\max \rho\left[T_{1}(u, v), T_{2} H(u, v)\right]=1,(u, v) \in \mathbb{P}_{1}$, for every choice of 
the topological mapping $H\left(\mathscr{P}_{1}\right)=\Phi_{2}$. Thus the relation $T_{1} \sim T_{2}(F)$ does not hold. Next let us choose $\mathcal{P}_{1}, \mathscr{P}_{2}, \mathcal{P}^{*}, T_{1}, T_{2}$ as follows.

$\mathbb{P}_{1}: 0 \leqq u \leqq 1,0 \leqq v \leqq 1 . \mathscr{P}_{2}: 0 \leqq \alpha \leqq 1,0 \leqq \beta \leqq 1 . \mathcal{P}^{*}: \xi^{2}+\eta^{2}+\zeta^{2}=1$.

We choose then $T_{1}$ as a monotone mapping from $\mathbb{P}_{1}$ onto $\mathcal{P}^{*}$ that carries the perimeter of $P_{1}$ into the single point $(0,0,1)$ of $P^{*}$, and we choose $T_{2}$ as a monotone mapping from $\mathbb{P}_{2}$ onto $\mathbb{P}^{*}$ that carries the perimeter of $\mathbb{P}_{2}$ into the single point $(0,0,-1)$ of $\mathbb{P}^{*}$. Thus on setting $M_{1}=T_{1}, M_{2}=T_{2}, \mathfrak{M}=\mathbb{P}^{*}$, and choosing $L$ as the identity, it is clear that $T_{1}, T_{2}$ satisfy the assumptions of statement (c) of 3.4. On the other hand, the relation $T_{1} \sim T_{2}(F)$ fails to hold. Indeed, let $H$ be any topological mapping from $\mathcal{P}_{1}$ onto $\mathcal{P}_{2}$, and let $\left(u_{0}, v_{0}\right)$ be any point on the perimeter of $\mathcal{P}_{1}$. Then $H\left(u_{0}, v_{0}\right)$ is a point on the perimeter of $P_{2}$, and hence $T_{1}\left(u_{0}, v_{0}\right)=(0,0,1), T_{2} H\left(u_{0}, v_{0}\right)=(0,0,-1)$, $\rho\left[T_{1}\left(u_{0}, v_{0}\right), T_{2} H\left(u_{0}, v_{0}\right)\right]=2$. Since $H$ was any topological mapping from $\mathscr{P}_{1}$ onto $\mathcal{P}_{2}$, it is now clear that the relation $T_{1} \sim T_{2}(F)$ does not hold.

3.6. We proceed to verify statement (b) in 3.4. Given $T_{1}, T_{2}$ as in 3.3, the middle-space $\mathfrak{M}$ occurring in the factorizations $3.3(1), 2.3(2)$ is by assumption a 2-cell. Since $L$ is continuous on $\mathfrak{M}$, for given $\epsilon>0$ we have an $\eta>0$ such that

$$
\rho\left[L\left(\bar{x}^{\prime}\right), L\left(\bar{x}^{\prime \prime}\right)\right]<\epsilon / 2 \quad \text { for } \quad \bar{x}^{\prime}, \bar{x}^{\prime \prime} \in \mathfrak{M}, \quad \rho\left(\bar{x}^{\prime}, \bar{x}^{\prime \prime}\right)<\eta .
$$

By 2.17 we have topological mappings $H_{1}\left(\mathscr{P}_{1}\right)=\mathfrak{M}, H_{2}\left(\mathscr{P}_{2}\right)=\mathfrak{M}$ such that

$$
\begin{array}{lll}
\rho\left[H_{1}\left(x_{1}\right), M_{1}\left(x_{1}\right)\right]<\eta & \text { for } & x_{1} \in \mathcal{P}_{1}, \\
\rho\left[H_{2}\left(x_{2}\right), M_{2}\left(x_{2}\right)\right]<\eta & \text { for } & x_{2} \in \mathcal{P}_{2} .
\end{array}
$$

Consider the topological mapping $H\left(\mathscr{P}_{1}\right)=\mathbb{P}_{2}$ defined by

$$
H\left(x_{1}\right)=H_{2}^{-1} H_{1}\left(x_{1}\right),
$$

We have then $\rho\left[T_{1}\left(x_{1}\right), T_{2} H\left(x_{1}\right)\right]=\rho\left[L M_{1}\left(x_{1}\right), L M_{2} H\left(x_{1}\right)\right] \leqq \rho\left[L M_{1}\left(x_{1}\right), L H_{1}\left(x_{1}\right)\right]$ $+\rho\left[L H_{1}\left(x_{1}\right), L M_{2} H\left(x_{1}\right)\right]$. Hence, by (1), (2)

$$
\rho\left[T_{1}\left(x_{1}\right), T_{2} H\left(x_{1}\right)\right]<\epsilon / 2+\rho\left[L H_{1}\left(x_{1}\right), L M_{2} H\left(x_{1}\right)\right], \quad x_{1} \in \mathcal{P}_{1} .
$$

Let us put $H\left(x_{1}\right)=x_{2}$. Then $H_{2}\left(x_{2}\right)=H_{2} H\left(x_{1}\right)=H_{1}\left(x_{1}\right)$ by (4), and hence, by (3),

$$
\rho\left[H_{1}\left(x_{1}\right), M_{2} H\left(x_{1}\right)\right]=\rho\left[H_{2}\left(x_{2}\right), M_{2}\left(x_{2}\right)\right]<\eta .
$$

By (1) it follows that

$$
\rho\left[L H_{1}\left(x_{1}\right), L M_{2} H\left(x_{1}\right)\right]<\epsilon / 2, \quad x_{1} \in \mathcal{P}_{1} .
$$

(5), (6) yield $\rho\left[T_{1}\left(x_{1}\right), T_{2} H\left(x_{1}\right)\right]<\epsilon$ for $x_{1} \in \mathcal{P}_{1}$. Since $\epsilon$ was arbitrary, the relation $T_{1} \sim T_{2}(F)$ is established.

3.7. As regards statement (c) in 3.4, let us first note that if the middlespace $\mathfrak{M}$ occurring in the factorizations $3.3(1), 3.3(2)$ is a 2 -sphere, then by 
2.3 the set $M_{1}\left(\mathscr{P}_{1}-\mathscr{P}_{1}^{0}\right)$ reduces to a single point $\bar{y}^{1}$ of $\mathfrak{M}$, and similarly the set $M_{2}\left(\mathscr{P}_{2}-\mathscr{P}_{2}^{0}\right)$ reduces to a single point $\bar{y}^{2}$ of $\mathfrak{M}$. If $\bar{y}^{1} \neq \bar{y}^{2}$, then the reasoning employed at the end of 3.5 shows that the relation $T_{1} \sim T_{2}(F)$ does not generally hold.

Lemma. Suppose that $T_{1}, T_{2}$, given as in 3.1, admit of simultaneous factorizations of the form

$$
\begin{array}{lll}
T_{1}=L M_{1}, & M_{1}\left(\mathscr{P}_{1}\right)=\mathfrak{M}, & L(\mathfrak{M})=\mathbb{P}^{*}, \\
T_{2}=L M_{2}, & M_{2}\left(\mathscr{P}_{2}\right)=\mathfrak{M}, & L(\mathfrak{M})=\mathscr{P}^{*},
\end{array}
$$

where the middle-space $\mathfrak{M}$ is a 2-sphere. Then the sets $M_{1}\left(\mathscr{P}_{1}-\mathbb{P}_{1}^{0}\right), M_{2}\left(\mathscr{P}_{2}-\mathbb{P}_{2}^{0}\right)$ reduce to single points $\bar{y}^{1}, \bar{y}^{2}$ of $\mathfrak{M}$, and the relation $T_{1} \sim T_{2}(F)$ holds if $\bar{y}^{1}=\bar{y}^{2}$.

Proof. Let us assume that $\bar{y}^{1}=\bar{y}^{2}=\bar{a}_{0}$. Take now any $\epsilon>0$. Since $L$ is continuous on $\mathfrak{M}$, we have then an $\eta>0$ such that

$$
\rho\left[L\left(\bar{y}^{\prime}\right), L\left(\bar{y}^{\prime \prime}\right)\right]<\epsilon \quad \text { if } \quad \bar{y}^{\prime}, \bar{y}^{\prime \prime} \in \mathfrak{M}, \rho\left(\bar{y}^{\prime}, \bar{y}^{\prime \prime}\right)<\eta .
$$

By 2.15 we have then a monotone mapping $\bar{M}_{1}\left(\mathscr{P}_{1}\right)=\mathfrak{M}$ with the following properties: (i) $\bar{M}_{1}\left(\mathscr{P}_{1}-\mathscr{P}_{1}^{0}\right)=\bar{a}_{0}$. (ii) $\rho\left[\bar{M}_{1}\left(x_{1}\right), M_{1}\left(x_{1}\right)\right]<\eta / 3$ for $x_{1} \in \mathscr{P}_{1}$. (iii) $\bar{M}_{1}$ maps $\mathcal{P}_{1}-M_{1}^{-1}\left(\bar{a}_{0}\right)$ topologically upon $\mathfrak{M}-\bar{a}_{0}$. Similarly, we have a monotone mapping $\bar{M}_{2}\left(\mathscr{P}_{2}\right)=\mathfrak{M}$ that has the same properties with respect to $M_{2}$. Now let $\bar{C}$ be a simple closed curve on $\mathfrak{M}$ that does not pass through $\bar{a}_{0}$. Then $\bar{C}$ determines two 2-cells $\bar{A}, \bar{B}$ on $\mathfrak{M}$, where the notation is so chosen that $\bar{a}_{0} \in \bar{A}$. Clearly, we can choose $\bar{C}$ so as to have $d(\bar{A})<\eta / 3$. Let us put

$$
\bar{M}_{1}^{-1}(\bar{B})=B_{1}, \quad \bar{M}_{2}^{-1}(\bar{B})=B_{2} .
$$

Then $B_{1}$ is a 2 -cell contained in $\mathbb{P}_{1}^{0}$, and $B_{2}$ is a 2 -cell contained in $\mathscr{P}_{2}^{0}$. Let us now consider the mapping $H\left(x_{1}\right)=\bar{M}_{2}^{-1} \bar{M}_{1}\left(x_{1}\right), x_{1} \in B_{1}$. Then $H$ is a topological mapping from $B_{1}$ onto $B_{2}$, and hence $H$ can be extended to a topological mapping from $\mathscr{P}_{1}$ onto $\mathcal{P}_{2}$ (cf. Newman [8, pp. 165-169]). The extended mapping $H$ possesses then the following properties.

$$
\begin{gathered}
H\left(\mathscr{P}_{1}\right)=\mathcal{P}_{2}, \quad H\left(B_{1}\right)=B_{2}, \quad H\left(\mathscr{P}_{1}-B_{1}\right)=\mathscr{P}_{2}-B_{2}, \\
H\left(x_{1}\right)=\bar{M}_{2}^{-1} \bar{M}_{1}\left(x_{1}\right) \quad \text { for } \quad x_{1} \in B_{1} .
\end{gathered}
$$

We assert that

$$
\rho\left[T_{1}\left(x_{1}\right), T_{2} H\left(x_{1}\right)\right]<\epsilon \text { for } x_{1} \in \mathcal{P}_{1} .
$$

Case (1). $x_{1} \in B_{1}$. Let us put $H\left(x_{1}\right)=x_{2}$. Then $x_{2} \in B_{2}$ and $x_{2}=\bar{M}_{2}^{-1} \bar{M}_{1}\left(x_{1}\right)$ by (3), (4). Since $\bar{M}_{1}, \bar{M}_{2}$ are bi-unique in $B_{1}, B_{2}$ respectively, it follows that $\bar{M}_{2}\left(x_{2}\right)=\bar{M}_{1}\left(x_{1}\right)$, and hence, by the choice of $\bar{M}_{2}$,

$$
\rho\left[\bar{M}_{1}\left(x_{1}\right), M_{2} H\left(x_{1}\right)\right]=\rho\left[\bar{M}_{2}\left(x_{2}\right), M_{2}\left(x_{2}\right)\right]<\eta / 3 .
$$


It follows that

$$
\begin{aligned}
\rho\left[M_{1}\left(x_{1}, M_{2} H\left(x_{1}\right)\right] \leqq\right. & \rho\left[M_{1}\left(x_{1}\right), \bar{M}_{1}\left(x_{1}\right)\right] \\
& +\rho\left[\bar{M}_{1}\left(x_{1}\right), M_{2} H\left(x_{1}\right)\right]<\eta / 3+\eta / 3 .
\end{aligned}
$$

In view of (1) we obtain finally

$$
\rho\left[T_{1}\left(x_{1}\right), T_{2} H\left(x_{1}\right)\right]=\rho\left[L M_{1}\left(x_{1}\right), L M_{2} H\left(x_{1}\right)\right]<\epsilon .
$$

Case (2). $x_{1} \in \mathcal{P}_{1}-B_{1}$. Then $x_{2}=H\left(x_{1}\right) \in \mathcal{P}_{2}-B_{2}$ by (3), and hence $\bar{M}_{1}\left(x_{1}\right)$ $\in \bar{A}^{0}, \bar{M}_{2} H\left(x_{1}\right) \in \bar{A}^{0}$ by (2). Consequently $\rho\left[\bar{M}_{1}\left(x_{1}\right), \bar{M}_{2} H\left(x_{1}\right)\right]<d(\bar{A})<\eta / 3$. It follows that

$$
\begin{aligned}
\rho\left[M_{1}\left(x_{1}\right), M_{2} H\left(x_{1}\right)\right] \leqq & \rho\left[M_{1}\left(x_{1}\right), \bar{M}_{1}\left(x_{1}\right)\right]+\rho\left[\bar{M}_{1}\left(x_{1}\right), \bar{M}_{2} H\left(x_{1}\right)\right] \\
& +\rho\left[\bar{M}_{2} H\left(x_{1}\right), M_{2} H\left(x_{1}\right)\right]<\eta / 3+\eta / 3+\eta / 3 .
\end{aligned}
$$

Hence, by (1), $\rho\left[T_{1}\left(x_{1}\right), T_{2} H\left(x_{1}\right)\right]=\rho\left[L M_{1}\left(x_{1}\right), L M_{2} H\left(x_{1}\right)\right]<\epsilon$.

Thus (5) is established. Since $\epsilon$ was arbitrary, the relation $T_{1} \sim T_{2}(F)$ is proved.

3.8. Let $T(\mathscr{P})=\mathbb{P}^{*}$ be a continuous mapping, where $P$ is a 2 -cell, and let

$$
T=L M, \quad M(\mathscr{P})=\mathfrak{M}, \quad L(\mathfrak{M})=\mathbb{P}^{*}
$$

be a monotone-light factorization of $T$. In view of the results in 3.6, 3.7, the case when $\mathfrak{M}$ is either a 2 -cell or a 2 -sphere is of particular importance. The mapping $T$ will be termed simple if $\mathfrak{M}$ is either a 2-cell or a 2-sphere (note that by 1.5 this property is independent of the particular choice of the monotone-light factorization). Let $K$ be the collection of maximal model continua determined by $T$ in $P$, and let $\gamma$ be a generic notation for a continuum $\gamma \in K$. The following special cases are of importance.

$(\alpha)$ Every $\gamma \in K$ reduces to a single point (in other words, $T$ is light). By 2.5 , it follows that the middle-space $\mathfrak{M}$ is a 2-cell, and hence $T$ is simple.

$(\beta)$ The perimeter of $P$ is a maximal model continuum, and every other maximal model continuum reduces to a single point. By 2.5 it follows that the middle space $\mathfrak{M}$ is a 2 -sphere, and hence $T$ is simple.

A mapping $T(\mathcal{P})=\mathbb{P}^{*}$, where $\mathcal{P}$ is a 2 -cell, will be termed a standard mapping if either $(\alpha)$ or $(\beta)$ holds.

3.9. Lemma. Let $Q$ denote the unit square $0 \leqq u \leqq 1,0 \leqq v \leqq 1$. If the mapping $T(P)=\Phi^{*}$, where $P$ is any 2 -cell, is simple, then there exists a standard simple mapping $\bar{T}(Q)=$ P* $^{*}$ such that $\bar{T} \sim T(F)$ (cf. 3.8).

Proof. Let $T=L M, M(\mathscr{P})=\mathfrak{M}, L(\mathfrak{M})=\mathscr{P}^{*}$ be a monotone-light factorization of $T$. By assumption, $\mathfrak{M}$ is either a 2-cell or a 2-sphere.

Case (1). $\mathfrak{M}$ is a 2-cell. Then there exists a topological mapping $H(Q)=\mathfrak{M}$. Let us put $\mathcal{P}_{1}=\mathscr{P}, M_{1}=M, T_{1}=T, \mathbb{P}_{2}=Q, M_{2}=H, T_{2}=L H$. Then $T_{1}, T_{2}$ satisfy the assumptions of statement (b) in 3.4, and hence $T_{2} \sim T_{1}(F)$ by 3.6. 
Since $T_{2}$ is clearly light, it follows that $\bar{T}=T_{2}$ satisfies our requirements.

Case (2). $\mathfrak{M}$ is a 2 -sphere. By 2.3 the mapping $M$ carries the perimeter of $\Phi$ into a single point $\bar{y}_{0}$ of $\mathfrak{M}$. Clearly, there exists a monotone mapping $\bar{M}(Q)=\mathfrak{M}$ that carries the perimeter of $Q$ into $\bar{y}_{0}$ and maps $Q^{0}$ topologically upon $\mathfrak{M}-\bar{y}_{0}$. On setting $\mathscr{P}_{1}=\mathscr{P}, M_{1}=M, T_{1}=T, \mathbb{P}_{2}=Q, M_{2}=\bar{M}, T_{2}=L \bar{M}$, the assumptions of the lemma in 3.7 are clearly satisfied, and hence $T_{2} \sim T_{1}(F)$. On the other hand, $(\beta)$ in 3.8 clearly holds for $T_{2}$. Thus $\bar{T}=T_{2}$ satisfies our requirements.

3.10. Let $T(\mathscr{P})=\mathbb{P}^{*}$, where $\mathcal{P}$ is a 2 -cell, be a continuous mapping. Suppose that the cyclic decomposition $\Delta$ of $T$ is nonvacuous, and let $T^{1}, \cdots$, $T^{n}, \ldots$ be the (finite or infinite) sequence of mappings comprised in $\Delta$. Then for each $T^{n}$ there exists a standard simple mapping $\bar{T}^{n}$ from the unit square $Q: 0 \leqq u \leqq 1,0 \leqq v \leqq 1$ onto the set $T^{n}(\mathcal{P})$ such that $\bar{T}^{n} \sim T^{n}(F)$ (cf. 1.9, 3.8).

Proof. Let $T=L M, M(\mathbb{P})=\mathfrak{M}, L(\mathfrak{M})=\mathbb{P}^{*}$ be a monotone-light factorization of $T$. By assumption, $\mathfrak{M}$ is not a dendrite. Let $\Gamma^{1}, \cdots, \Gamma^{n}, \cdots$ be the (finite or infinite) sequence of the proper cyclic elements of $\mathfrak{M}$. For each $n$, let $\mu^{n}$ be the (unique) monotone retraction from $\mathfrak{M}$ onto $\Gamma^{n}$. Then the cyclic decomposition of $T$ consists of the transformations $T^{1}=L \mu^{1} M, \cdots, T^{n}=L \mu^{n} M, \cdots$ (see 1.9). Now since $\mathcal{P}$ is a 2-cell, $\Gamma^{n}$ is either a 2-cell or a 2 -sphere (see 2.3). On the other hand, since $M$ and $\mu^{n}$ are both monotone, we have for $T^{n}$ the monotone-light factorization $T^{n}=L M^{n}$, where $M^{n}=\mu^{n} M$, with the middlespace $\mathfrak{M}^{n}=\mu^{n} M(\mathscr{P})=\mu^{n}(\mathfrak{M})=\Gamma^{n}$. Thus $T^{n}$ is simple, and the existence of $\bar{T}^{n}$, with the required properties, follows directly from 3.9.

3.11. Given $T_{1}, T_{2}$ as in 3.1, suppose that $T_{1} \sim T_{2}(F)$. By 3.2 we have then simultaneous factorizations of the form 3.2 (1), $3.2(2)$, with the same middlespace $\mathfrak{M}$ and the same light factor $L$. Let us assume that $\mathfrak{M}$ is not a dendrite. Let $\Gamma^{1}, \cdots, \Gamma^{n}, \cdots$ be the proper cyclic elements of $\mathfrak{M}$, and let $\mu^{n}(\mathfrak{M})=\Gamma^{n}$ be the (unique) monotone retraction from $\mathfrak{M}$ onto $\Gamma^{n}$. Then we have (see 1.9) for the cyclic decompositions $\Delta_{1}, \Delta_{2}$ of $T_{1}, T_{2}$ respectively the expressions

$$
\Delta_{1}=\left[L \mu^{1} M_{1}, \cdots, L \mu^{n} M_{1}, \cdots\right], \quad \Delta_{2}=\left[L \mu^{1} M_{2}, \cdots, L \mu^{n} M_{2}, \cdots\right] .
$$

According to 1.11 , the assumption $T_{1} \sim T_{2}(F)$ implies that $\Delta_{1} \sim \Delta_{2}(F)$, and under the present circumstances there may be a temptation to expect that $L \mu^{n} M_{1} \sim L \mu^{n} M_{2}(F)$ for every $n$. Simple examples show that this is not necessarily the case. As a matter of fact, the result in 1.11, applied to the present situation, merely asserts that there exists a rearrangement $L \bar{\mu}^{1} M_{2}, \cdots$, $L \bar{\mu}^{n} M_{2}, \cdots$ of the sequence $L \mu^{1} M_{2}, \cdots, L \mu^{n} M_{2}, \cdots$, such that $L \mu^{n} M_{1}$ $\sim L \bar{\mu}^{n} M_{2}(F)$ for every $n$.

\section{Additivity theorems}

4.1. In the sequel, $P$ will denote a fixed Peano space, $P^{*}$ a fixed metric space (in the applications, $P^{*}$ coincides with Euclidean three-space). $\mathcal{T}$ will denote the class of all continuous mappings from $P$ into $P^{*}$ (that is, $T(\mathcal{P})$ 
$=P^{*} \subset P^{*}$. Let us observe that $\mathscr{P}$ and $P^{*}$ are fixed spaces, while $\mathscr{P}^{*}=T(\mathcal{P})$ depends upon $T$. The image space $\mathscr{P}^{*}=T(\mathcal{P})$ may reduce to a single point. This will happen if and only if $T$ is constant on $P$.

If $T_{1}(\mathcal{P})=\mathcal{P}_{1}^{*}, T_{2}(\mathcal{P})=\mathcal{P}_{2}^{*}$ are two mappings in $\mathcal{T}$, then we define

$$
\rho\left[T_{1}, T_{2}\right]=\max \rho\left[T_{1}(x), T_{2}(x)\right], \quad x \in \mathcal{P},
$$

where the use of the maximum is justified since $\mathcal{P}$ is compact and $T_{1}, T_{2}$ are continuous. If $T, T_{n} \in \mathcal{T}, n=1,2, \cdots$, and $\rho\left[T, T_{n}\right] \rightarrow 0$ for $n \rightarrow \infty$, then we shall write $T_{n} \rightarrow T$.

If $T \in \mathcal{T}$, and $T=L M, M(\mathscr{P})=\mathfrak{M}, L(\mathfrak{M})=\mathscr{P}^{*}$ is a monotone-light factorization of $T$, then the middle-space $\mathfrak{M}$ is determined by $T$ up to a homeomorphism (see 1.5). Thus if $\mathfrak{M}$ turns out to be a simple arc, for example, for one monotone-light factorization of $T$, then the same will be the case for every monotone-light factorization of $T$. In view of this situation, we can speak of the middle-space being a single point, or a dendrite, or a single cyclic chain, and so forth, without specifying a definite monotone-light factorization.

4.2. Let $T \in \mathcal{T}$. The cyclic decomposition $\Delta(T)$ of $T$ (see 1.9) is independent of the choice of a monotone-light factorization for $T$. On the other hand, we shall have to operate with conceptions that do depend upon the choice of the factorization, and special care will be needed in such cases. Let $T \in \mathcal{T}$, and let

$$
T=L M, \quad M(\mathcal{P})=\mathfrak{M}, \quad L(\mathfrak{M})=\mathbb{P}^{*}
$$

be a definite monotone-light factorization of $T$. Let $\bar{A}$ be an $A$-set of $\mathfrak{M}$ (note that $\mathfrak{M}$ is a Peano space). To avoid trivial discussions, let us assume that $\mathfrak{M}$ does not reduce to a single point, and let us agree that we consider only $A$-sets that do not reduce to single points. Now there exists a unique monotone retraction $\alpha(\mathfrak{M})=\bar{A}$. In terms of $\alpha$, we define the transformation $L \alpha M$ which we shall denote by $T \mid \bar{A}$. Since $\alpha M$ is a monotone mapping from $\mathcal{P}$ onto $\bar{A}$, we have for $T \mid \bar{A}$ the monotone-light factorization

$$
T \mid \bar{A}=L(\alpha M), \quad \alpha M(\mathcal{P})=\bar{A} .
$$

Clearly, $T \mid \bar{A}$ maps $P$ onto a subset of $\mathcal{P}^{*}=T(\mathscr{P})$, and hence $T \mid \bar{A} \in \mathcal{T}$. Thus we associate, in terms of a fixed factorization (1), with each $A$-set $\bar{A}$ of $\mathfrak{M}$ a mapping $T \mid \bar{A}$ together with a definite monotone-light factorization (2) of $T \mid \bar{A}$. Let now $\bar{A}_{1}, \bar{A}_{2}$ be two $A$-sets in $\mathfrak{M}$, such that $\bar{A}_{2} \subset \bar{A}_{1}$. We have then the formula

$$
\left(T \mid \bar{A}_{1}\right)\left|\bar{A}_{2}=T\right| \bar{A}_{2} \text {. }
$$

Indeed, let $\alpha_{1}(\mathfrak{M})=\bar{A}_{1}, \alpha_{2}(\mathfrak{M})=\bar{A}_{2}$ be the monotone retractions corresponding to $\bar{A}_{1}, \bar{A}_{2}$ respectively. By definition

$$
T \mid \bar{A}_{1}=L \alpha_{1} M, \quad \alpha_{1} M(P)=\bar{A}_{1},
$$




$$
T \mid \bar{A}_{2}=L \alpha_{2} M, \quad \alpha_{2} M(P)=\bar{A}_{2} .
$$

Now $\bar{A}_{1}$ is a Peano subspace of $\mathfrak{M}$, and $\bar{A}_{2}$ is an $A$-set relative to $\bar{A}_{1}$ also. Thus the symbol $\left(T \mid \bar{A}_{1}\right) \mid \bar{A}_{2}$ is meaningful, and in view of the factorization (4) assigned to $T \mid \bar{A}_{1}$, we have the formula

$$
\left(T \mid \bar{A}_{1}\right) \mid \bar{A}_{2}=L \alpha_{12} \alpha_{1} M,
$$

where $\alpha_{12}\left(\bar{A}_{1}\right)=\bar{A}_{2}$ is the unique monotone retraction from $\bar{A}_{1}$ onto $\bar{A}_{2}$. Then $\alpha_{12} \alpha_{1}$ and $\alpha_{2}$ are both monotone retractions from $\mathfrak{M}$ onto $\bar{A}_{2}$, and since there exists just one such monotone retraction, we must have $\alpha_{12} \alpha_{1}=\alpha_{2}$. Thus (3) follows from (5) and (6).

If $\mathfrak{M}$ is not a dendrite, and if $\Gamma^{1}, \cdots, \Gamma^{n}, \ldots$ are the proper cyclic elements of $\mathfrak{M}$, then each $\Gamma^{n}$ is an $A$-set of $\mathfrak{M}$, and hence we can consider the mapping $T \mid \Gamma^{n}$. Comparison with 1.9 yields the formula

$$
\Delta(T)=\left[T\left|\Gamma^{1}, \cdots, T\right| \Gamma^{n}, \cdots\right] .
$$

Let $\bar{A}_{1}, \cdots, \bar{A}_{n}, \cdots$ be a (finite or countably infinite) sequence of $A$-sets in $\mathfrak{M}$, such that $\mathfrak{M}=\bar{A}_{1}+\cdots+\bar{A}_{n}+\cdots$, and $\left(\bar{A}_{1}+\cdots+\bar{A}_{n}\right) \bar{A}_{n+1}=\bar{a}_{n}$ $=$ single point of $\mathfrak{M}$. Then each $\bar{A}_{n}$ is a Peano space, and the proper cyclic elements of $\bar{A}_{n}$ coincide with those proper cyclic elements of $\mathfrak{M}$ that are subsets of $\bar{A}_{n}$. Furthermore, each proper cyclic element of $\mathfrak{M}$ is a subset of exactly one $\bar{A}_{n}$. Now let $\Gamma$ be a proper cyclic element of $\mathfrak{M}$ and let $\Gamma \subset \bar{A}_{n}$. Then $\Gamma$ contributes to $\Delta(T)$ the mapping $T \mid \Gamma$ and to $\Delta\left(T \mid \bar{A}_{n}\right)$ the mapping $\left(T \mid \bar{A}_{n}\right)|\Gamma=T| \Gamma$ (see (3)). Thus each $\Gamma$ contributes the same mapping to $\Delta(T)$ and to $\Delta\left(T \mid \bar{A}_{n}\right)$, where $\bar{A}_{n}$ is the (unique) term of the sequence $\bar{A}_{1}, \cdots, \bar{A}_{n}, \cdots$ that contains $\Gamma$. There follows the formula

$$
\Delta(T)=\Delta\left(T \mid \bar{A}_{1}\right)+\cdots+\Delta\left(T \mid \bar{A}_{n}\right)+\cdots .
$$

If $\mathfrak{M}$ is a dendrite, then the same formula holds, since then all the cyclic decompositions involved are vacuous.

Let us now consider an approximation to $\mathfrak{M}$ in terms of a sequence of cyclic chains. According to the cyclic chain approximation theorem, we can choose in $\mathfrak{M}$ a (finite or countably infinite) sequence of cyclic chains $\bar{C}_{1}, \cdots$, $\bar{C}_{n}, \cdots$, such that the following holds (see Whyburn $[6$, p. 73 , Theorem 7.1]):

(a) $\left(\bar{C}_{1}+\cdots+\bar{C}_{n}\right) \bar{C}_{n+1}$ is a single point for every $n$.

(b) Setting $H_{n}=\bar{C}_{1}+\cdots+\bar{C}_{n}$, each $H_{n}$ is an $A$-set. If the sequence $\bar{C}_{n}$ is infinite, then the maximum diameter of the components of $\mathfrak{M}-H_{n}$ converges to zero for $n \rightarrow \infty$, and the diameter of $\bar{C}_{n}$ approaches zero.

(c) Setting $H=\bar{C}_{1}+\cdots+\bar{C}_{n}+\cdots$, every point in $\mathfrak{M}-H$ is an end point of $\mathfrak{M}$.

It follows that every proper cyclic element is a subset of precisely one of the cyclic chains $\bar{C}_{1}, \cdots, \bar{C}_{n}, \cdots$. Thus the reasoning used to derive (8) 
applies (even though $\bar{C}_{1}+\cdots+\bar{C}_{n}+\cdots$ may be different from $\mathfrak{M}$ ), and there follows the formula

$$
\Delta(T)=\Delta\left(T \mid \bar{C}_{1}\right)+\cdots+\Delta\left(T \mid \bar{C}_{n}\right)+\cdots .
$$

We assumed in this section that $\mathfrak{M}$ does not reduce to a single point. If $\mathfrak{M}$ does reduce to a single point, then our statements become vacuously true.

4.3. Let there be given a function $F(T)$, defined for every $T \in \mathcal{T}$, with the following properties.

(i) For given $T \in \mathcal{T}, F(T)$ is a non-negative real number, possibly equal to $+\infty$.

(ii) $F(T)$ is lower semi-continuous. That is, if $T, T_{n} \in \mathcal{T}$ and $T_{r} \rightarrow T$ (cf. 4.1), then $F(T) \leqq \lim \inf F\left(T_{n}\right)$.

Let $T \in \mathcal{T}$, and let $\Delta(T)$ be the cyclic decomposition of $T$. We define a quantity $F[\Delta(T)]$ as follows. If $\Delta(T)=0$, then we put $F[\Delta(T)]=0$. If $\Delta(T) \neq 0$, then $\Delta(T)=\left[T^{1}, \cdots, T^{n}, \cdots\right]$, where each $T^{n} \in \mathcal{T}$. If the series $F\left(T^{1}\right)+\cdots+F\left(T^{n}\right)+\cdots$ converges, then $F[\Delta(T)]$ is defined as the sum of this series. If the series diverges, then we put $F[\Delta(T)]=+\infty$. Note that $F[\Delta(T)]$ is independent of the arrangement of the sequence $T^{n}$, since $F$ is non-negative.

4.4. We shall study conditions for the formula $F(T)=F[\Delta(T)]$ to hold for every $T \in \mathcal{T}$. If this is the case, then $F(T)$ will be termed cyclicly additive. The additional conditions placed upon $F(T)$, to enforce cyclic additivity, will be chosen with certain applications in mind, and may seem unmotivated and unnecessarily involved at first sight. Granting that simpler and equally useful conditions may be discovered in the future, we shall first derive some necessary conditions for cyclic additivity, and we shall study some of the mutual implications between these conditions. In fact, this part of our discussion will prove useful in producing a compact proof for our main result. It also serves as a motivation for the conditions used to secure cyclic additivity.

4.5. Assume that $F(T)$, given as in 4.3 , is cyclicly additive. If the middlespace $\mathfrak{M}$ for a $T \in \mathcal{T}$ is a dendrite, then $\Delta(T)=0$, and hence $F(T)=F[\Delta(T)]$ $=0$. Let is note the following special cases.

(iii) If the middle-space $\mathfrak{M}$ for a $T \in \mathcal{T}$ reduces to a single point, then $F(T)=0$. Alternatively, if $T$ is constant, then $F(T)=0$.

(iv) If the middle-space $\mathfrak{M}$ for a $T \in \mathcal{T}$ is a simple arc, then $F(T)=0$.

4.6. More involved implications of cyclic additivity may be stated in terms of a monotone-light factorization $T=L M, M(\mathbb{P})=\mathfrak{M}, L(\mathfrak{M})=\mathbb{P}^{*}$, where $T \in \mathcal{T}$. It is assumed that $F(T)$, given as in 4.3 , is cyclicly additive.

(v) Let $\bar{A}_{0}$ be an $A$-set in $\mathfrak{M}$ such that $\mathfrak{M}-\bar{A}_{0} \neq 0$, and let $\bar{S}_{1}, \cdots$, $\bar{S}_{n}, \cdots$ be the (finite or countably infinite) sequence of the components of $\mathfrak{M}-\bar{A}_{0}$. Then the closure $c\left(\bar{S}_{n}\right)$ of $\bar{S}_{n}$ is an $A$-set and

$$
F(T)=F\left(T \mid \bar{A}_{0}\right)+F\left[T \mid c\left(\bar{S}_{1}\right)\right]+\cdots+F\left[T \mid c\left(\bar{S}_{n}\right)\right]+\cdots
$$


(vi) Let $\bar{A}_{1}, \bar{A}_{2}$ be (nondegenerate) $A$-sets in $\mathfrak{M}$ such that $\bar{A}_{1}+\bar{A}_{2}=\mathfrak{M}$, and $\bar{A}_{1} \bar{A}_{2}$ is a single point. Then

$$
F(T)=F\left(T \mid \bar{A}_{1}\right)+F\left(T \mid \bar{A}_{2}\right) .
$$

(vii) Let $\bar{A}_{1}, \cdots, \bar{A}_{n}, \cdots$ be a (finite or countably infinite) sequence of nondegenerate $A$-sets, such that $\bar{A}_{1}+\cdots+\bar{A}_{n}+\cdots=\mathfrak{M}$, and for each $n$ the product $\left(\bar{A}_{1}+\cdots+\bar{A}_{n}\right) \bar{A}_{n+1}$ reduces to a single point. Then

$$
F(T)=F\left(T \mid \bar{A}_{1}\right)+\cdots+F\left(T \mid \bar{A}_{n}\right)+\cdots .
$$

(viii) Let $\bar{a}_{0}$ be a cut point of $\mathfrak{M}$, and let $\bar{S}_{1}, \cdots, \bar{S}_{n}, \cdots$ be the (finite or countably infinite) sequence of the components of $\mathfrak{M}-\bar{a}_{0}$. Then for each $n$, the closure $c\left(\bar{S}_{n}\right)$ of $\bar{S}_{n}$ is an $A$-set, and

$$
F(T)=F\left[T \mid c\left(\bar{S}_{1}\right)\right]+\cdots+F\left[T \mid c\left(\bar{S}_{n}\right)\right]+\cdots \cdot
$$

(ix) Let $\bar{A}_{1}, \cdots, \bar{A}_{n}, \cdots$ be a (finite or countably infinite) sequence of nondegenerate $A$-sets such that for every $n$ the product $\left(\bar{A}_{1}+\cdots+\bar{A}_{n}\right) \bar{A}_{n+1}$ is either empty or else reduces to a single point. Then

$$
F(T) \geqq F\left(T \mid \bar{A}_{1}\right)+\cdots+F\left(T \mid \bar{A}_{n}\right)+\cdots .
$$

(x) Let $\bar{C}_{1}, \cdots, \bar{C}_{n}, \cdots$ be a sequence of cyclic chains in $\mathfrak{M}$ that approximate to $\mathfrak{M}$ in the sense of the cyclic chain approximation theorem (see 4.2). Then

$$
F(T)=F\left(T \mid \bar{C}_{1}\right)+\cdots+F\left(T \mid \bar{C}_{n}\right)+\cdots \cdot
$$

Proof. Clearly, (v), (vi), (viii) are special cases of (vii). To prove (vii), note that by $4.2(8)$ we have $\Delta(T)=\Delta\left(T \mid \bar{A}_{1}\right)+\cdots+\Delta\left(T \mid \bar{A}_{n}\right)+\cdots \cdot$ Hence (see the introduction), $F[\Delta(T)]=F\left[\Delta\left(T \mid \bar{A}_{1}\right)\right]+\cdots+F\left[\Delta\left(T \mid \bar{A}_{n}\right)\right]$ $+\cdots$, and (vii) follows in view of the cyclic additivity of $F$. In a similar way, (x) follows from 4.2 (9). The proof of (ix) is equally immediate.

4.7. Given $F(T)$ as in 4.3 , we shall say that $F$ possesses the property (vii), say, if the preceding statement (vii) holds for every $T \in \mathcal{T}$, and the properties (i), (ii), . . (x) are defined in the same way. A further relevant, and unfortunately not simple, property will now be introduced. Let $T \in \mathcal{T}$, and let $\gamma$ be a continuum in $P$ such that $T$ is constant on $\gamma$ and $P-\gamma \neq 0$. Thus $T(\gamma)=a_{0}{ }^{*}$ is a single point of the space $\mathbb{P}^{*}=T(\mathcal{P})$, and $\gamma \subset T^{-1}\left(a_{0}^{*}\right)$. It is not assumed that $\gamma$ is a maximal model continuum. Let $D$ be a component of $P-\gamma$. Let us define the mappings $T_{1}, T_{2}$ as follows

$$
T_{1}(x)=\left\{\begin{array}{l}
T(x) \text { in } D, \\
a_{0}^{*} \text { in } P-D,
\end{array} \quad T_{2}(x)=\left\{\begin{array}{l}
T(x) \text { in } P-D, \\
a_{0}^{*} \text { in } D .
\end{array}\right.\right.
$$

Clearly $T_{1}, T_{2} \in \mathcal{T}$. If $F(T)=F\left(T_{1}\right)+F\left(T_{2}\right)$ for every choice of $T, \gamma, D$ with the properties just stated, then we shall say that $F$ is additive with respect to 
continua of constancy. In the way of motivation, let us state that this property is available in the applications, and that it is a necessary condition for cyclic additivity. This latter assertion will be verified presently.

4.8. Suppose that $F$, given as in 4.3 , is cyclicly additive. Then $F$ is additive with respect to continua of constancy.

Proof. Let $T, \gamma, D, a_{0}{ }^{*}, T_{1}, T_{2}$ be given as in 4.7. Since $\mathcal{P}$ is a Peano space, we have the inclusion

$$
\text { fr }(D) \subset \gamma,
$$

where $\operatorname{fr}(D)$ denotes the frontier of $D$.

Case (1). $T$ is constant in $D$. Then $T(x)=a_{0}^{*}$ for $x \in D$ in view of (1). Hence $T_{1}$ is constant and $T_{2}=T$. By 4.5 (iii) it follows that $F\left(T_{1}\right)+F\left(T_{2}\right)$ $=0+F(T)=F(T)$.

Case (2). $T$ is constant in $P-D$. Then $T(x)=a_{0}{ }^{*}$ for $x \in \mathcal{P}-D$, since $\gamma \subset \mathcal{P}-D$. Thus $T_{1}=T$ and $T_{2}$ is constant. The formula $F(T)=F\left(T_{1}\right)+F\left(T_{2}\right)$ follows as in case (1).

Case (3). $T$ is not constant either in $D$ or in $P-D$. Let $T=L M, M(\mathcal{P})=\mathfrak{M}$, $L(\mathfrak{M})=\mathbb{P}^{*}$ be a monotone-light factorization of $T$. By assumption $T(\gamma)$ $=L M(\gamma)=a_{0}^{*}$, and hence $M(\gamma) \subset L^{-1}\left(a_{0}^{*}\right)$. Since $M(\gamma)$ is connected and $L^{-1}\left(a_{0}^{*}\right)$ is totally disconnected, it follows that $M(\gamma)$ is a single point $\bar{a}_{0}$ of $\mathfrak{M}$, such that $L\left(\bar{a}_{0}\right)=a_{0}{ }^{*}$. We proceed to verify the following auxiliary statements.

(a) Let $\bar{C}$ be a component of $\mathfrak{M}-\bar{a}_{0}$. We assert that either $M^{-1}(\bar{C}) \subset D$ or else $M^{-1}(\bar{C}) \subset P-D$. Indeed, if this were not the case, then the continuum $M^{-1}(\bar{C})$ would intersect both $D$ and $\Phi-D$, and hence $M^{-1}(\bar{C})$ would intersect $\mathrm{fr}(D)$. In view of (1) it would follow that $\gamma M^{-1}(\bar{C}) \neq 0$, while $\gamma M^{-1}(\bar{C})$ $\subset M^{-1}\left(\bar{a}_{0}\right) M^{-1}(\bar{C}) \subset M^{-1}\left(\bar{a}_{0}\right) M^{-1}\left(\mathfrak{M}-\bar{a}_{0}\right)=0$.

(b) $\mathfrak{M}-\bar{a}_{0} \neq 0$. Indeed, $T$ is not constant.

(c) $\mathfrak{M}-\bar{a}_{0}$ is disconnected. Indeed, if $\mathfrak{M}-\bar{a}_{0}$ is connected, then $M^{-1}\left(\mathfrak{M}-\bar{a}_{0}\right)$ is either a subset of $D$ or a subset of $P-D$, by (a). In the first case, it would follow that $T$ is constant on $P-D$, and in the second case it would follow that $T$ is constant on $D$, in contradiction with our present assumptions.

(d) Let $\bar{B}_{1}$ be the sum of those components of $\mathfrak{M}-\bar{a}_{0}$ that intersect $M(D)$, and let $\bar{B}_{2}$ be the sum of those components of $\mathfrak{M}-\bar{a}_{0}$ that intersect $M(P-D)$. Then $\bar{B}_{1} \neq 0$ since $T$ is not constant on $D, \bar{B}_{2} \neq 0$ since $T$ is not constant on $\mathbb{P}-D, \quad \bar{B}_{1} \bar{B}_{2}=0$ by (a), and clearly $\bar{B}_{1}+\bar{B}_{2}=\mathfrak{M}-\bar{a}_{0}$. Obviously $M(D)$ $\subset \bar{B}_{1}+\bar{a}_{0}, M(P-D) \subset \bar{B}_{2}+\bar{a}_{0}$.

Now let us put $\bar{A}_{1}=\bar{B}_{1}+\bar{a}_{0}, \bar{A}_{2}=\bar{B}_{2}+\bar{a}_{0}$. Since $\bar{B}_{1}, \bar{B}_{2}$ are sums of components of $\mathfrak{M}-\bar{a}_{0}, \bar{A}_{1}$ and $\bar{A}_{2}$ are $A$-sets, and by (d)

$$
\bar{A}_{1}+\bar{A}_{2}=\mathfrak{M}, \quad \bar{A}_{1} \bar{A}_{2}=\bar{a}_{0} .
$$

Let us note that $\bar{A}_{1}, \bar{A}_{2}$ are nondegenerate $A$-sets. Indeed, $\bar{A}_{1}-\bar{a}_{0}=\bar{B}_{1} \neq 0$, $\bar{A}_{2}-\bar{a}_{0}=\bar{B}_{2} \neq 0$ by (d). Let us now consider the monotone retractions $\alpha_{1}(\mathfrak{M})=\bar{A}_{1}, \alpha_{2}(\mathfrak{M})=\bar{A}_{2}$. Since $M(D) \subset \bar{A}_{1}, M(\mathscr{P}-D) \subset \bar{A}_{2}$ by (d), we have 


$$
\begin{aligned}
& \alpha_{1} M(x)=M(x) \quad \text { if } \quad x \in D \text {, } \\
& \alpha_{1} M(x)=\bar{a}_{0} \quad \text { if } \quad x \in P-D, \\
& \alpha_{2} M(x)=\bar{a}_{0} \quad \text { if } \quad x \in D \text {, } \\
& \alpha_{2} M(x)=M(x) \quad \text { if } \quad x \in P-D \text {. }
\end{aligned}
$$

Comparison with $4.7,4.2$ yields $T_{1}=T\left|\bar{A}_{1}, T_{2}=T\right| \bar{A}_{2}$. Hence $F(T)=F\left(T_{1}\right)$ $+F\left(T_{2}\right)$ by (2) and 4.6 (vi).

4.9. Suppose that $F$, given as in 4.3 , possesses the property (v) (see 4.7, 4.6). Then $F$ also possesses the properties (vi) to (x).

Proof of property (viii) (see 4.6). Put $\bar{A}_{0}=\bar{S}_{1}+\bar{a}_{0}$. Then $\bar{A}_{0}$ is an $A$-set, and the components of $\mathfrak{M}-\bar{A}_{0}$ are precisely $\bar{S}_{2}, \cdots, \bar{S}_{n}, \cdots$. Furthermore, $\bar{A}_{0}=c\left(\bar{S}_{1}\right)$. Thus (viii) appears as a direct consequence of $(\mathrm{v})$.

Proof of property (vi) (see 4.6). Let $\bar{S}_{1}^{\prime}, \cdots, \bar{S}_{n}^{\prime}, \ldots$ be the components of $\mathfrak{M}-\bar{A}_{1}$, and let $\bar{a}_{n}^{\prime}$ be the unique frontier point of $\bar{S}_{n}^{\prime}$. Then $\bar{a}_{n}^{\prime} \in \bar{A}_{1}$, and since $\bar{S}_{n}^{\prime} \subset \bar{A}_{2}$, we have also $\bar{a}_{r}^{\prime} \in \bar{A}_{2}$. As $\bar{A}_{1} \bar{A}_{2}=\bar{a}_{0}$, it follows that $\bar{a}_{n}^{\prime}=\bar{a}_{0}$ for every $n$. Since $\bar{A}_{1}$ is an $A$-set, each $\bar{S}_{n}^{\prime}$ is a component of $\mathfrak{M}-\bar{a}_{n}^{\prime}=\mathfrak{M}-\bar{a}_{0}$. Thus $\bar{A}_{2}-\bar{a}_{0}=\bar{S}_{1}^{\prime}+\cdots+\bar{S}_{n}^{\prime}+\cdots$ is a sum of components of $\mathfrak{M}-\bar{a}_{0}$, and similarly $\bar{A}_{1}-\bar{a}_{0}$ is a sum of components of $\mathfrak{M}-\bar{a}_{0}$. Hence by (viii), applied to the mappings $T\left|\bar{A}_{1}, T\right| \bar{A}_{2}, T$ respectively, we obtain

$$
\begin{aligned}
F\left(T \mid \bar{A}_{2}\right)= & F\left[\left(T \mid \bar{A}_{2}\right) \mid c\left(\bar{S}_{1}^{\prime}\right)\right]+\cdots+F\left[\left(T \mid \bar{A}_{2}\right) \mid c\left(\bar{S}_{n}^{\prime}\right)\right]+\cdots, \\
F\left(T \mid \bar{A}_{1}\right)= & \left.F\left[T \mid \bar{A}_{1}\right) \mid c\left(\bar{S}_{1}^{\prime \prime}\right)\right]+\cdots+F\left[\left(T \mid \bar{A}_{1}\right) \mid c\left(\bar{S}_{m}^{\prime \prime}\right)\right]+\cdots, \\
F(T)= & \left\{F\left[T \mid c\left(\bar{S}_{1}^{\prime}\right)\right]+\cdots+F\left[T \mid c\left(\bar{S}_{n}^{\prime}\right)\right]+\cdots\right\} \\
& +\left\{F\left[T \mid c\left(\bar{S}_{1}^{\prime \prime}\right)\right]+\cdots+F\left[T \mid c\left(\bar{S}_{m}^{\prime \prime}\right)\right]+\cdots\right\},
\end{aligned}
$$

where $\bar{S}_{1}^{\prime \prime}, \cdots, \bar{S}_{m}^{\prime \prime}, \cdots$ are the components of $\mathfrak{M}-\bar{A}_{2}$. In view of 4.2 (3) the formula $F(T)=F\left(T \mid \bar{A}_{1}\right)+F\left(T \mid \bar{A}_{2}\right)$ follows.

Proof of property (vii) (see 4.6). Case (1). The given sequence of $A$-sets is a finite sequence $\bar{A}_{1}, \cdots, \bar{A}_{n}$. For $n=1$, we have $\bar{A}_{1}=\mathfrak{M}, T\left|\bar{A}_{1}=T\right| \mathfrak{M}=T$ and the formula $F(T)=F\left(T \mid \bar{A}_{1}\right)$ is obvious. Assume that property (vii) has been shown to hold for finite sequences of $A$-sets with not more than $n-1$ terms. Then for a sequence $\bar{A}_{1}, \cdots, \bar{A}_{n}$ with $n$ terms we put $\bar{A}_{1}^{\prime}=\bar{A}_{1}+\cdots$ $+\bar{A}_{n-1}, \bar{A}_{2}^{\prime}=\bar{A}_{n}$. By property (vi), already established, we have then (cf. 4.2 (3))

$$
\begin{aligned}
F(T)= & \left.F\left(T \mid \bar{A}_{1}^{\prime}\right)+F\left(T \mid \bar{A}_{2}^{\prime}\right)=F\left[T \mid \bar{A}_{1}^{\prime}\right) \mid \bar{A}_{1}\right]+\cdots \\
& +F\left[\left(T \mid \bar{A}_{1}^{\prime}\right) \mid \bar{A}_{n-1}\right]+F\left(T \mid \bar{A}_{n}\right)=F\left(T \mid \bar{A}_{1}\right)+\cdots+F\left(T \mid \bar{A}_{n}\right) .
\end{aligned}
$$

Case (2). The sequence $\bar{A}_{1}, \cdots, \bar{A}_{n}, \cdots$ is infinite. Put $\bar{A}_{1}+\cdots+\bar{A}_{n}$ $=\bar{A}_{n}^{\prime}$, and let $d_{n}^{\prime}$ denote the maximum diameter of the components of $\mathfrak{M}-\bar{A}_{n}^{\prime}$. We have then the relations

$$
d_{n}^{\prime} \rightarrow 0, \quad d\left(\bar{A}_{n}\right) \rightarrow 0
$$

for $n \rightarrow \infty$.

The simple proofs are analogous to those used in establishing the cyclic chain approximation theorem, and are left to the reader (cf. Whyburn [6, pp. 73- 
74]). Now let $\alpha_{n}^{\prime}$ be the (unique) monotone retraction from $\mathfrak{M}$ onto the $A$-set $\bar{A}_{n}^{\prime}$. Clearly $\rho\left[M(x), \alpha_{n}^{\prime} M(x)\right] \leqq d_{n}^{\prime}$ for every point $x \in \mathcal{P}$. Thus $\alpha_{n}^{\prime} M$ converges uniformly to $M$, and hence $T \mid \bar{A}_{n}^{\prime}=L \alpha_{n}^{\prime} M$ converges uniformly to $L M=T$. By 4.3 (ii) there follows the inequality

$$
F(T) \leqq \lim \inf F\left(T \mid \bar{A}_{n}^{\prime}\right),
$$$$
n \rightarrow \infty \text {. }
$$

Now by case (1), applied to $F\left(T \mid \bar{A}_{n}{ }^{\prime}\right)$, we have (cf. 4.2 (3))

$$
F\left(T \mid \bar{A}_{n}^{\prime}\right)=F\left(T \mid \bar{A}_{1}\right)+\cdots+F\left(T \mid \bar{A}_{n}\right) .
$$

(2) and (3) yield the inequality

$$
F(T) \leqq F\left(T \mid \bar{A}_{1}\right)+\cdots+F\left(T \mid \bar{A}_{n}\right)+\cdots \cdot
$$

Now observe that by the (presupposed) property (v) we have $F\left(T \mid \bar{A}_{0}\right)$ $\leqq F(T)$ for every $A$-set $\bar{A}_{0}$ in $\mathfrak{M}$, since $F$ is non-negative. Hence in particular

$$
F\left(T \mid \bar{A}_{n}^{\prime}\right) \leqq F(T), \quad n=1,2, \cdots .
$$

(3), (4), (5) yield $F(T)=F\left(T \mid \bar{A}_{1}\right)+\cdots+F\left(T \mid \bar{A}_{n}\right)+\cdots$.

Proof of property (ix) (see 4.6). Case (1). The given sequence of $A$-sets is finite. Let then $\bar{A}_{1}, \cdots, \bar{A}_{n}$ be the sequence. If $n=1$, then by the (presupposed) property (v) we have $F\left(T \mid \bar{A}_{1}\right) \leqq F(T)$, since $F$ is non-negative. Proceeding by induction, let us assume that (ix) is established for finite sequences with not more than $n-1$ terms. Given then a sequence $\bar{A}_{1}, \cdots, \bar{A}_{n}$, suppose first that these sets are disjoint. Let $\bar{S}_{1}, \ldots, \bar{S}_{k}, \ldots$ be the components of $\mathfrak{M}-\bar{A}_{n}$. Since each $\bar{A}_{i}$ is connected and $\bar{A}_{i} \subset \mathfrak{M}-\bar{A}_{n}$ for $\mathrm{i}=1, \cdots$, $n-1$, it follows that $\bar{A}_{i}$ is a subset of one of the sets $\bar{S}_{1}, \ldots, \bar{S}_{k}, \ldots$ Let us put

$$
\Sigma_{k}=\Sigma F\left(T \mid \bar{A}_{i}\right), \quad \bar{A}_{i} \subset \bar{S}_{k} .
$$

Of course, this summation may be vacuous for a given $k$, and then $\Sigma_{k}=0$. Now for each $k$ the summation contains at most $n-1$ terms, and hence $\Sigma_{k} \leqq F\left[T \mid c\left(\bar{S}_{k}\right)\right]$ (cf. $4.2(3)$ ). On the other hand, we have by property (v)

$$
F(T)=F\left(T \mid \bar{A}_{n}\right)+F\left[T \mid c\left(\bar{S}_{1}\right)\right]+\cdots+F\left[T \mid c\left(\bar{S}_{k}\right)\right]+\cdots
$$

and the inequality $F(T) \geqq F\left(T \mid \bar{A}_{1}\right)+\cdots+F\left(T \mid \bar{A}_{n}\right)$ follows. Suppose, secondly, that the sets $\bar{A}_{1}, \cdots, \bar{A}_{n}$ are not disjoint, say $\bar{A}_{1} \bar{A}_{2} \neq 0$. Then $\bar{A}_{1} \bar{A}_{2}$ is a single point, and hence $\bar{A}_{1}+\bar{A}_{2}$ is again an $A$-set. We have therefore the inequality

$$
F(T) \geqq F\left[T \mid\left(\bar{A}_{1}+\bar{A}_{2}\right)\right]+F\left(T \mid \bar{A}_{3}\right)+\cdots+F\left(T \mid \bar{A}_{n}\right) .
$$

In view of property (vi), already established, the inequality $F(T) \geqq F\left(T \mid \bar{A}_{1}\right)$ $+\cdots+F\left(T \mid \bar{A}_{n}\right.$ ) follows (cf. $4.2(3)$ ).

Case (2). The sequence $\bar{A}_{1}, \cdots, \bar{A}_{n}, \cdots$ is infinite. For each $n$ we have then $F(T) \geqq F\left(T \mid \bar{A}_{1}\right)+\cdots+F\left(T \mid \bar{A}_{n}\right)$ by case (1), and for $n \rightarrow \infty$ the desired inequality follows. 
Proof of property (x). By property (ix), just established, we have the inequality

$$
F(T) \geqq F\left(T \mid \bar{C}_{1}\right)+\cdots+F\left(T \mid \bar{C}_{n}\right)+\cdots .
$$

To establish the complementary inequality, it is sufficient to consider the case when the sequence $\bar{C}_{1}, \cdots, \bar{C}_{n}, \cdots$ is infinite, since in the finite case the desired result follows directly from property (vii). Setting $H_{n}=\bar{C}_{1}+\cdots$ $+\bar{C}_{n}$, let $d_{n}^{\prime}$ denote the maximum diameter of the components of $\mathfrak{M}-H_{n}$. We have then $d_{n}^{\prime} \rightarrow 0$ (see 4.2). Since $H_{n}$ is an $A$-set, we have a unique monotone retraction $\beta_{n}(\mathfrak{M})=H_{n}$. Clearly $\rho\left[M(x), \beta_{n} M(x)\right] \leqq d_{n}^{\prime}$ for every point $x \in \mathcal{P}$. Thus $\beta_{n} M$ converges uniformly to $M$, and hence $T \mid H_{n}=L \beta_{n} M$ converges uniformly to $T=L M$. Hence (see 4.3 (ii)),

$$
F(T) \leqq \lim \inf F\left(T \mid H_{n}\right),
$$$$
n \rightarrow \infty \text {. }
$$

On the other hand, by 4.2 (3) and property (vii) (already established) we have

$$
F\left(T \mid H_{n}\right)=F\left(T \mid \bar{C}_{1}\right)+\cdots+F\left(T \mid \bar{C}_{n}\right) .
$$

(6), (7), (8) yield the desired formula for $F(T)$.

4.10. Suppose that $F$, given as in 4.3 , is additive with respect to continua of constancy (see 4.7). Then $F$ possesses the properties (v) to (x) (see 4.7, 4.6).

Proof. In view of 4.9 it is sufficient to verify property $(\mathrm{v})$. Using the notations $T, L, M, \mathfrak{M}, \bar{A}_{0}, \bar{S}_{1}, \cdots, \bar{S}_{n}, \cdots$ in the sense of $4.6(\mathrm{v})$, we make the proof in the following steps.

Case (1). The set $\mathfrak{M}-\bar{A}_{0}$ has exactly one component $\bar{S}_{1}$. Then the frontier of $\bar{S}_{1}$ consists of a single point $\bar{a}_{0} \in \bar{A}_{0}$, and $\bar{S}_{1}$ is a component of $\mathfrak{M}-\bar{a}_{0}$. Hence, on setting $\gamma=M^{-1}\left(\bar{a}_{0}\right), D=M^{-1}\left(\bar{S}_{1}\right), D$ is a component of $P-\gamma$. Let $\alpha$ be the monotone retraction from $\mathfrak{M}$ onto $\bar{A}_{0}$, and let $\beta$ be the monotone retraction from $\mathfrak{M}$ onto $c\left(\bar{S}_{1}\right)=\bar{S}_{1}+\bar{a}_{0}$ (note that $c\left(\bar{S}_{1}\right)$ is an $A$-set). We have then

$$
\begin{array}{llll}
\alpha M(x)=\bar{a}_{0} & \text { for } \quad x \in D, & & \alpha M(x)=M(x) \text { for } x \in \mathcal{P}-D, \\
\beta M(x)=M(x) & \text { for } \quad x \in D, & & \beta M(x)=\bar{a}_{0} \text { for } x \in \mathcal{P}-D .
\end{array}
$$

Setting $a_{0}^{*}=L\left(\bar{a}_{0}\right)$, it follows that $T(\gamma)=L M(\gamma)=L\left(\bar{a}_{0}\right)=a_{0}^{*}$, and

$$
L \alpha M(x)=\left\{\begin{array}{l}
a_{0}^{*} \text { in } D, \\
T(x) \text { in } P-D,
\end{array} \quad L \beta M(x)=\left\{\begin{array}{l}
T(x) \text { in } D, \\
a_{0}^{*} \text { in } \mathcal{P}-D .
\end{array}\right.\right.
$$

Thus $L \beta M=T_{1}, L \alpha M=T_{2}$, where $T_{1}, T_{2}$ are defined as in 4.7. Since $F$ is additive with respect to continua of constancy, we have therefore (cf. 4.2)

$$
F(T)=F\left(T_{1}\right)+F\left(T_{2}\right)=F(L \alpha M)+F(L \beta M)=F\left(T \mid \bar{A}_{0}\right)+F\left(T \mid \bar{S}_{1}\right) .
$$

Case (2). $\mathfrak{M}-\bar{A}_{0}$ has a finite number of components $\bar{S}_{1}, \cdots, \bar{S}_{n}$. Since the case $n=1$ has been already taken care of, we can proceed by induction. So 
let us assume that $(\mathrm{v})$ is already established for the case when the number of components $\bar{S}$ does not exceed $n-1$. On setting $\bar{A}_{0}^{\prime}=\bar{A}_{0}+\bar{S}_{2}+\cdots+\bar{S}_{n}$, $\mathfrak{M}-\bar{A}_{0}^{\prime}$ has the single component $\bar{S}_{1}$, and hence by case (1)

$$
F(T)=F\left(T \mid \bar{A}_{0}^{\prime}\right)+F\left[T \mid c\left(\bar{S}_{1}\right)\right] .
$$

On the other hand, the components of $\bar{A}_{0}^{\prime}-\bar{A}_{0}$ are $\bar{S}_{2}, \cdots, \bar{S}_{n}$, and hence property (v) is available for the mapping $T \mid \bar{A}_{0}^{\prime}$ and the $A$-set $\bar{A}_{0}$. Therefore (cf. $4.2(3)$ )

$$
F\left(T \mid \bar{A}_{0}^{\prime}\right)=F\left(T \mid \bar{A}_{0}\right)+F\left[T \mid c\left(\bar{S}_{2}\right)\right]+\cdots+F\left[T \mid c\left(\bar{S}_{n}\right)\right] .
$$

(1) and (2) yield $F(T)=F\left(T \mid \bar{A}_{0}\right)+F\left[T \mid c\left(\bar{S}_{1}\right)\right]+\cdots+F\left[T \mid c\left(\bar{S}_{n}\right)\right]$.

Case (3). The sequence of components $\bar{S}_{1}, \cdots, \bar{S}_{n}, \cdots$ of $\mathfrak{M}-\bar{A}_{0}$ is infinite. Then $d\left(\bar{S}_{n}\right) \rightarrow 0$ and hence also

$$
d\left[c\left(\bar{S}_{n}\right)\right] \rightarrow 0
$$

for $n \rightarrow \infty$.

Let us put $\bar{A}_{n}=\bar{A}_{0}+c\left(\bar{S}_{1}\right)+\cdots+c\left(\bar{S}_{n}\right)$. Then $\bar{A}_{n}$ is an $A$-set. If $\alpha_{n}$ is the monotone retraction from $\mathfrak{M}$ onto $\bar{A}_{n}$, then clearly $\alpha_{n} M$ converges uniformly to $M$ as a consequence of (3), and hence $T \mid \bar{A}_{n}=L \alpha_{n} M$ converges uniformly to $T=L M$. By 4.3 (ii) it follows that

$$
F(T) \leqq \lim \inf F\left(T \mid \bar{A}_{n}\right), \quad n \rightarrow \infty .
$$

On the other hand, by case (2) we have (cf. $4.2(3)$ )

$$
F\left(T \mid \bar{A}_{n}\right)=F\left(T \mid \bar{A}_{0}\right)+F\left[T \mid c\left(\bar{S}_{1}\right)\right]+\cdots+F\left[T \mid c\left(\bar{S}_{n}\right)\right] .
$$

(4) and (5) yield the inequality

$$
F(T) \leqq F\left(T \mid \bar{A}_{0}\right)+F\left[T \mid c\left(\bar{S}_{1}\right)\right]+\cdots+F\left[T \mid c\left(\bar{S}_{n}\right)\right]+\cdots .
$$

Let us put now $\bar{A}_{n}^{\prime}=\bar{A}_{0}+\bar{S}_{n+1}+\bar{S}_{n+2}+\cdots$. Then $\bar{A}_{n}^{\prime}$ is again an $A$-set. If $\alpha_{n}^{\prime}$ denotes the monotone retraction from $\mathfrak{M}$ onto $\bar{A}_{n}{ }^{\prime}$, then in view of (3) clearly $\alpha_{n}^{\prime}$ converges uniformly to the monotone retraction $\alpha_{0}(\mathfrak{M})=\bar{A}_{0}$, and hence $T \mid \bar{A}_{n}^{\prime}=L \alpha_{n}^{\prime} M$ converges uniformly to $T \mid \bar{A}_{0}=L \alpha_{0} M$. By 4.3 (ii) it follows that

$$
F\left(T \mid \bar{A}_{0}\right) \leqq \lim \inf F\left(T \mid \bar{A}_{n}^{\prime}\right),
$$$$
n \rightarrow \infty \text {. }
$$

On the other hand, since $\mathfrak{M}-\bar{A}_{n}^{\prime}$ has a finite number of components (namely $\bar{S}_{1}, \cdots, \bar{S}_{n}$ ), we have by case (2)

$$
F(T)=F\left(T \mid \bar{A}_{n}^{\prime}\right)+F\left[T \mid c\left(\bar{S}_{1}\right)\right]+\cdots+F\left[T \mid c\left(\bar{S}_{n}\right)\right] .
$$

(7) and (8) yield the inequality

$$
F(T) \geqq F\left(T \mid \bar{A}_{0}\right)+F\left[T \mid c\left(\bar{S}_{1}\right)\right]+\cdots+F\left[T \mid c\left(\bar{S}_{n}\right)\right]+\cdots \cdot
$$

(6) and (9) yield property (v) (see 4.6, 4.7). 
4.11. To proceed further, it seems necessary to restrict the space $P$. In the principal applications at this time, $P$ is either a 2-cell or a 2 -sphere, but it may be of interest to point out the relevant property that enables us to deal with these special cases simultaneously.

If $\mathfrak{M}$ is a Peano space, and if every simple arc $\alpha \subset \mathfrak{M}$ is a monotone retract of $\mathfrak{M}$, then we shall say that $\mathfrak{M}$ possesses the property $(\pi)$. Clearly this property is cyclicly extensible. If $\mathscr{P}$ is a Peano space, and if for every continuous monotone mapping $M(\mathscr{P})=\mathfrak{M}$ the image space $\mathfrak{M}$ possesses the property $(\pi)$, then we shall say that $P$ possesses the property (II). Choosing $M$ as the identity, we see that the property (II) implies the property $(\pi)$. The following remarks will be useful in the sequel.

(a) Suppose that $P$ possesses the property (I). Let $M(P)=\mathfrak{M}$ be a continuous monotone mapping, and let $\bar{C}$ be a proper cyclic element of $\mathfrak{M}$. Then $\bar{C}$ possesses the property $(\pi)$. Indeed, using the monotone retraction $\mu(\mathfrak{M})=\bar{C}$, we see that $\mu M(P)=\bar{C}$ is a continuous monotone mapping from $P$ onto $\bar{C}$, and the assertion follows.

(b) Suppose that $\mathcal{P}$ possesses the property $(\Pi)$, and let $M(\mathscr{P})=\mathfrak{M}$ be a continuous monotone mapping. Let $\bar{a}_{1}, \bar{a}_{2}$ be two distinct points of $\mathfrak{M}$, and suppose that the cyclic chain $\bar{C}\left(\bar{a}_{1}, \bar{a}_{2}\right)$ does not reduce to a simple arc. Let $\bar{C}_{1}, \cdots, \bar{C}_{n}, \cdots$ be the proper cyclic elements of $\mathfrak{M}$ that occur in $\bar{C}\left(\bar{a}_{1}, \bar{a}_{2}\right)$, and let $\gamma$ be a simple arc with end points $\bar{a}_{1}, \bar{a}_{2}$. Then $\gamma \subset \bar{C}\left(\bar{a}_{1}, \bar{a}_{2}\right)$, and for each $n$ the product $\gamma \bar{C}_{n}$ is a simple arc $\gamma_{n}$. Furthermore, the simple arcs $\gamma_{1}, \cdots, \gamma_{n}, \cdots$ have no interior points in common. Now let $k$ be any positive integer. We assert the existence of a monotone retraction $\mu_{k}\left[\bar{C}\left(\bar{a}_{1}, \bar{a}_{2}\right)\right]=\gamma+\bar{C}_{1}+\cdots+\bar{C}_{k}$. To obtain $\mu_{k}$, note that by (a) above the simple arc $\gamma_{n}$ is a monotone retract of $\bar{C}_{n}$. Let $\alpha_{n}\left(\bar{C}_{n}\right)=\gamma_{n}$ be a monotone retraction. Let $\mu_{k}$ coincide with the identity on $\gamma+\bar{C}_{1}+\cdots+\bar{C}_{k}$ and with $\alpha_{n}$ on $\bar{C}_{n}$ for $n>k$. Then clearly $\mu_{k}$ has the desired properties. The continuity of $\mu_{k}$ follows from the fact that $d\left(\bar{C}_{n}\right) \rightarrow 0$ for $n \rightarrow \infty$. This same fact implies that $\mu_{k}$ converges uniformly to the identity for $k \rightarrow \infty$.

(c) Obviously, the 2 -sphere and the 2 -cell possess the property $(\pi)$. It follows that they also possess the property (II). Indeed, if $P$ is either the 2-sphere or the 2-cell, and if $M(\mathscr{P})=\mathfrak{M}$ is any continuous monotone mapping, then every proper cyclic element $\bar{C}$ of $\mathfrak{M}$ is either a 2-cell or a 2 -sphere. Thus every proper cyclic element of $\mathfrak{M}$ possesses the property $(\pi)$, and since this property is clearly cyclicly extensible, the assertion follows.

(d) Although implicitly contained in the preceding remarks, let us stress the fact that if $\mathfrak{M}$ is a dendrite, then $\mathfrak{M}$ possesses the property $(\pi)$. Indeed, if $\gamma$ is a simple arc in $\mathfrak{M}$, then $\gamma$ coincides in this case with the cyclic chain that joins its end points, and hence $\gamma$ is a monotone retract of $\mathfrak{M}$.

4.12. Cyclic additivity theorem. Let $\mathcal{T}$ be the class of continuous mappings from a fixed Peano space $P$ into a fixed metric space $P^{*}$ (that is, $T(\mathcal{P}) \subset P^{*}$ for every $T \in \mathcal{T}$ ). Let $F(T)$ be a real-valued function defined for $T \in \mathcal{T}$. Suppose that the following conditions hold. 
(a) $P$ possesses the property (II). In particular, $P$ may be either a 2 -cell or a 2 -sphere $\left(\right.$ see $(4.11)\left({ }^{3}\right)$.

(b) $F(T) \geqq 0$ for $T \in \mathcal{T}$. For certain mappings $T \in \mathcal{T}, F(T)$ may be equal to $+\infty$.

(c) $F(T)$ is lower semi-continuous (see 4.3).

(d) If $T$ is constant, then $F(T)=0$.

(e) If the middle-space occurring in the monotone-light factorization of a $T \in \mathcal{T}$ is a simple arc, then $F(T)=0$.

(f) $F(T)$ is additive with respect to continua of constancy (see 4.7). Then $F(T)$ is cyclicly additive (see 4.4 ).

Proof. By 4.10 it follows that $F(T)$ possesses all the properties (v) to (x) (cf. 4.6, 4.7). Hence, as a special case of property (ix), we have the inequality $F[\Delta(T)] \leqq F(T)$. Thus it is sufficient to prove the inequality

$$
F(T) \leqq F[\Delta(T)] .
$$

Let $T \in \mathcal{T}$, and let $T=L M, M(\mathscr{P})=\mathfrak{M}, L(\mathfrak{M})=\mathscr{P}^{*}$ be a monotone-light factorization of $T$.

Case (A). $\mathfrak{M}$ reduces to a single cyclic chain $\bar{C}\left(\bar{a}_{1}, \bar{a}_{2}\right)$. Let then $\gamma$ be a simple arc, with end points $\bar{a}_{1}, \bar{a}_{2}$, in $\mathfrak{M}$.

Subcase (A.1). $\mathfrak{M}=\gamma$. Then $\Delta(T)=0$ and hence $F[\Delta(T)]=0$. By condition (e), $F(T)=0$. Hence (1) holds.

Subcase (A.2). $\mathfrak{M}$ has a finite number of proper cyclic elements $\bar{C}_{1}, \cdots, \bar{C}_{n}$. In view of the structure of a cyclic chain, we have then a finite system $\bar{A}_{1}, \cdots, \bar{A}_{N}$ of $A$-sets in $\mathfrak{M}$, such that $\bar{A}_{1}+\cdots+\bar{A}_{N}=\mathfrak{M}$, $\left(\bar{A}_{1}+\cdots+\bar{A}_{k}\right) \bar{A}_{k+1}$ is a single point for $k=1, \cdots, N-1$, and each $\bar{A}_{k}$ is either a simple arc or a proper cyclic element. By property (vii) we have then $F(T)=F\left(T \mid \bar{A}_{1}\right)+\cdots+F\left(T \mid \bar{A}_{N}\right)$. Now if $\bar{A}_{k}$ is a simple arc for a certain $k$ then $F\left(T \mid \bar{A}_{k}\right)=0$ by condition (e), and hence (1) follows (in fact, directly with the sign of equality).

Subcase (A.3). $\mathfrak{M}$ has infinitely many proper cyclic elements $\bar{C}_{1}, \cdots$, $\bar{C}_{n}, \cdots$. Using the notations of $4.11(\mathrm{~b})$, consider the monotone retraction $\mu_{k}(\mathfrak{M})=\mu_{k}\left[\bar{C}\left(\bar{a}_{1}, \bar{a}_{2}\right)\right]=\gamma+\bar{C}_{1}+\cdots+\bar{C}_{k}$. Since $\mu_{k}$ converges uniformly to the identity on $\mathfrak{M}$, clearly the mapping $L \mu_{k} M$ converges uniformly to $L M=T$. Hence, by condition (c)

$$
F(T) \leqq \lim \inf F\left(L \mu_{k} M\right), \quad k \rightarrow \infty .
$$

On the other hand, the middle-space in the monotone light factorization $L \mu_{k} M=L\left(\mu_{k} M\right)$ coincides with the set $\gamma+\bar{C}_{1}+\cdots+\bar{C}_{k}$, and thus this middle-space is a cyclic chain with a finite number of proper cyclic elements. Hence, by subcase (A.2), $F\left(L \mu_{k} M\right) \leqq F\left[\Delta\left(L \mu_{k} M\right)\right]$. Now let $\beta_{i}$ be the monotone

$\left({ }^{3}\right)$ According to a recent communication, G. T. Whyburn has proved that (I) is equivalent to unicoherence for Peano spaces. 
retraction from $\gamma+\bar{C}_{1}+\cdots+\bar{C}_{k}$ onto $\bar{C}_{i}, i=1, \cdots, k$. Then

$$
\Delta\left(L \mu_{k} M\right)=\left[L \beta_{1} \mu_{k} M, \cdots, L \beta_{k} \mu_{k} M\right] .
$$

Now $\beta_{i} \mu_{k}$ is a monotone retraction from $\mathfrak{M}$ onto $\bar{C}_{i}, i=1, \cdots, k$. This monotone retraction being unique, it follows that $L \beta_{i} \mu_{k} M=T \mid \bar{C}_{i}, i=1, \cdots, k$. Hence $\Delta\left(L \mu_{k} M\right)=\left[T\left|\bar{C}_{1}, \cdots, T\right| \bar{C}_{k}\right]$, and consequently (cf. 4.3 and condition (b))

$$
F\left[\Delta\left(L \mu_{k} M\right)\right]=F\left(T \mid \bar{C}_{1}\right)+\cdots+F\left(T \mid \bar{C}_{k}\right) \leqq F[\Delta(T)] .
$$

(2) and (3) imply (1). Let us note that some caution had to be exercised since $\gamma+\bar{C}_{1}+\cdots+\bar{C}_{k}$ is not an $A$-set relative to $\mathfrak{M}$.

Case (B). $\mathfrak{M}$ does not reduce to a single cyclic chain. Let then $\bar{C}_{1}, \cdots$, $\bar{C}_{n}, \ldots$ be a sequence of cyclic chains in $\mathfrak{M}$ that approximate to $\mathfrak{M}$ in the sense of the cyclic chain approximation theorem (see 4.2). By 4.2 (9), we have then

$$
\Delta(T)=\Delta\left(T \mid \bar{C}_{1}\right)+\cdots+\Delta\left(T \mid \bar{C}_{n}\right)+\cdots,
$$

and hence (see the introduction)

$$
F[\Delta(T)]=F\left[\Delta\left(T \mid \bar{C}_{1}\right)\right]+\cdots+F\left[\Delta\left(T \mid \bar{C}_{n}\right)\right]+\cdots .
$$

On the other hand, the middle-space corresponding to $T \mid \bar{C}_{n}$ may be chosen as $\bar{C}_{n}$ (cf. 4.2). Hence, by case (A),

$$
F\left[\Delta\left(T \mid \bar{C}_{n}\right)\right] \geqq F\left(T \mid \bar{C}_{n}\right), \quad n=1,2, \cdots .
$$

By property (x) (see $4.7,4.10$ ), we have $F\left(T \mid \bar{C}_{1}\right)+\cdots+F\left(T \mid \bar{C}_{n}\right)+\cdots$ $=F(T)$. Thus (4) and (5) imply (1), and the proof is complete.

\section{BIBLIOGRAPHY}

1. H. Hahn, Reelle Funktionen, part 1, Leipzig, 1932.

2. B. v. Kerékjárt6, Involutions et surfaces continues, Acta Univ. Szeged. vol. 3 (1927) pp. 49-67.

3. C. B. Morrey, The topology of (path) surfaces, Amer. J. Math. vol. 57 (1935) pp. 17-50.

4. - An analytic characterization of surfaces of finite Lebesgue area, part 2, Amer. J. Math. vol. 58 (1936) pp. 313-322.

5. T. Rad6, On surface area, Proc. Nat. Acad. Sci. U.S.A. vol. 31 (1945) pp. 102-106.

6. G. T. Whyburn, Analytic topology, Amer. Math. Soc. Colloquium Publications, vol. 28, 1942.

7. J. W. T. Youngs, The topological theory of Fréchet surfaces, Ann. of Math. vol. 45 (1944) pp. 753-785.

8. M. H. A. Newman, Elements of the topology of plane sets of points, Cambridge University Press, 1939.

The Institute for Advanced Study,

Princeton, N. J.

The Ohio State University, Columbus, Ohio. 